\title{
Peces del Golfo de Nicoya, Pacífico, Costa Rica: actualización de las listas de los buques científicos Skimmer y Victor Hensen
}

\author{
José A. Vargas-Zamora ${ }^{1,3}$, Myrna I. López-Sánchez² \& Ana R. Ramírez-Coghi ${ }^{1,2}$ \\ 1. Escuela de Biología. Universidad de Costa Rica, 11501-2060, San José, Costa Rica; jose.vargas@ucr.ac.cr \\ 2. Museo de Zoología, Escuela de Biología, Universidad de Costa Rica, 11501-2060, San José, Costa Rica; \\ myrna.lopez@ucr.ac.cr; ana.ramirezcoghi@ucr.ac.cr \\ 3. Centro de Investigación en Ciencias del Mar y Limnología (CIMAR), Universidad de Costa Rica, 11501-2060, San \\ José, Costa Rica
}

Recibido 19-III-2019. C Corregido 12-VI-2019. Aceptado 08-VIII-2019.

\begin{abstract}
Fishes from the Gulf of Nicoya, Pacific, Costa Rica: an update of the lists of the Skimmer and Victor Hensen research vessels. Introduction: Since the middle of the $\mathrm{XX}^{\text {th }}$ century the Gulf of Nicoya estuary $\left(10^{\circ} \mathrm{N} \& 85^{\circ} \mathrm{W}\right)$ has been the main source of numerous fishing resources, such as mollusks, crustaceans, and fish. The estuary is presently under the increasing impact of local, regional and global stressors. The expeditions on board the Skimmer (1979-1980) and Victor Hensen (1993-1994) research vessels provided multidisciplinary data on the estuary in support of management policies. However, updated information is still needed. Objectives: To update the published lists of fish species from both expeditions and make suggestions for new research areas for future evaluations of the ecosystem. Methods: The fish collections by the Skimmer covered a depth range of 10 to $50 \mathrm{~m}$ using an otter trawl net, and those aboard the Victor Hensen were made between 10 to $228 \mathrm{~m}$ depth using otter and beam trawl nets. The identification of most of the fish species and the measurement of its lenghts were made on board of both vessels. The species names were updated based on recent literature and the web page World Register of Marine Species (WORMS). The list of species identified in recent years based on voucher specimens from the estuary and deposited in the Ichthyology Collection of the Museum of Zoology, University of Costa Rica, was also included. Results: The total number of identified species from both vessels is 268. A new species of Pontinus is under description. Of the total, 19 are cartilaginous fishes and 249 are bony fishes. The Sciaenidae and the flatfishes were represented by 31 and 32 species, respectivley. The flatfish genus Symphurus was the most diverse, with 11 species. Eighteen species were identified after the publication of the original lists. Eight species were described based on specimens collected during the expeditions: Gymnothorax phalarus, Notarius biffi, N. cookei, Ophicthus mecoptera, O. melope, Sphoeroides rosenblatti, Trinectes xanthurus and Urotrygon cimar. The five longer specimens collected by both vessels, were: the longtail sting ray Hypanus longus $(117 \mathrm{~cm})$, the pike conger Cynoponticus coniceps $(103 \mathrm{~cm})$, the brown sea catfish Sciades dowii $(87 \mathrm{~cm})$, the electric ray Narcine brancroftii $(80 \mathrm{~cm})$, and the queen corvina Cynoscion albus $(75 \mathrm{~cm})$. Conclusions: In view of the increasing number of users of the estuarine resources, it is urgent a multidisciplinary evaluation of the ecosystem in support of new management policies. The expected results may facilitate updating the Gulf of Nicoya Trophic Model, a management tool available for the estuary since 1998 and based mainly on information gathered during both expeditions. Ecosystem stressors, such as the extraction of resources, the input of pollutants and its possible link to endocrine disruption, as well as the presence of natural barriers such as hypoxia at deeper waters at the mouth of the estuary must be taken into account in a future evaluation.
\end{abstract}

Key words: estuarine fish; fisheries; fish lengths; trawl nets; benthos; Prionotus; Porichthys; Symphurus.

Vargas-Zamora, J. A., López-Sánchez, M. I. \& Ramírez-Coghi, A. R. (2019). Peces del Golfo de Nicoya, Pacífico, Costa Rica: actualización de las listas de los buques científicos Skimmer y Victor Hensen. Revista de Biología Tropical, 67(4), 913-934. 
Los ecosistemas costeros alrededor del mundo, especialmente los estuarios, han sido objeto de la alteración de su entorno y de la captura intensiva (sobrepesca) de sus recursos naturales, de los que los moluscos, crustáceos y peces son los más explotados (Gladstone, 2009). La sobrepesca de esos recursos ha sido la causa más evidente del colapso de muchas pesquerías alrededor del mundo (Jackson et al., 2001). La extracción excesiva no solo disminuye las poblaciones hasta densidades críticas, sino que también altera el flujo de energía del ecosistema, llevándolo a otros estados de funcionamiento no necesariamente útiles para el entorno humano (Cloern et al., 2016). En el Golfo de Nicoya la extracción ha sido intensiva, especialmente de especies filtradoras (Ej: moluscos bivalvos y sardinas), depredadoras (Ej: peces pargos y corvinas) o detritófagas (Ej: varios crustáceos). Especies de peces como Cynoscium albus (corvina reina, Sciaenidae) y Lutjanus colorado (pargo colorado, Lutjanidae) son muy apreciadas, entre otras, desde hace décadas, en el mercado local.

El Golfo de Nicoya es el principal ecosistema estuarino de Costa Rica y, desde mediados del siglo XX, en forma cada vez más intensa, soporta varias pesquerías que extraen manualmente algunos moluscos y peces, o emplean diferentes tipos de artes no selectivas para la captura de la fauna epibéntica, de la que se seleccionan preferentemente camarones y algunos peces, descartándose una gran parte del resto de la captura.

Entre 1979 y 1981 y con el propósito de aportar información para dar apoyo a la formulación de políticas de manejo del ecosistema del Golfo se ejecutó, con la participación del buque científico Skimmer, un programa de investigación multidisciplinario cuyos resultados han sido resumidos por Vargas (1995). Las evaluaciones de la diversidad, abundancia relativa y distribución espacial de los peces demersales del Golfo fueron incluidas en ese programa y realizadas en febrero y julio de 1979 y abril de 1980, con una red de arrastre (tipo otter) y en un ámbito de profundidad de 10 a $50 \mathrm{~m}$. La lista de los peces recolectados fue publicada por Bartels, Price, López y Bussing (1983) e incluyó 189 registros, de los que 32 quedaron provisionalmente a nivel de género y los restantes 157 a nivel de especie.

En diciembre de 1993 y febrero de 1994 se hizo una nueva evaluación multidisciplinaria del Golfo de Nicoya, así como de la región de Bahía Coronado y el Golfo Dulce, a bordo del buque científico Víctor Hensen (en adelante, V. Hensen). Parte de los resultados de esa expedición fueron recopilados por Vargas y Wolff (1996). Para la evaluación de los peces demersales se utilizaron dos tipos de redes (otter y beam) y se recolectaron muestras entre los 10 y los $228 \mathrm{~m}$ de profundidad en el Golfo de Nicoya. La lista de los peces fue publicada por Bussing y López (1996) y comprende, para el Golfo de Nicoya, 185 registros a nivel de especie y seis a nivel de género. La lista incluyó aparte las 20 especies recolectadas en las cuatro estaciones profundas (64 a $228 \mathrm{~m}$ ) de la boca del estuario, por lo que el total anotado para el Golfo en ese crucero ascendió a 205 especies y seis a nivel de género.

Las actividades pesqueras en el Golfo de Nicoya continúan actualmente a lo largo del estuario y, con redes de arrastre, a mayores profundidades (Herrera, Clarke, Naranjo-Elizondo, Espinoza, \& Wehrtmann, 2016). En este contexto se requieren alternativas actualizadas para el manejo de esos recursos y estas deben estar fundamentadas en una nueva evaluación de las especies sobrevivientes y sus parámetros poblacionales. Para lograr este objetivo es necesaria una base de datos actualizada sobre las especies de peces encontradas previamente en el estuario, su frecuencia en las estaciones y las longitudes de los ejemplares recolectados. En este contexto, la información publicada proveniente de los buques Skimmer y V. Hensen, por su ámbito de profundidad y artes utilizadas, es la más amplia disponible sobre los peces capturados con redes de arrastre en el Golfo de Nicoya. La información más reciente, sobre las especies de peces de la región interna del Golfo de Nicoya, fue publicada por Murase, Angulo, Miyasaki, Bussing y López (2014), con base en los registros desde 1962 del Museo de Zoología 
de la Universidad de Costa Rica, e incluyó 274 especies.

Por tanto, el objetivo de este estudio es proveer un inventario actualizado de las especies de peces identificados durante, o posteriormente, provenientes de las expediciones de los buques Skimmer y V. Hensen, frecuencia de algunas especies en las estaciones, así como sugerencias para futuras evaluaciones del ecosistema.

\section{MATERIALES Y MÉTODOS}

Las capturas de peces en los ambientes sedimentarios del fondo del Golfo de Nicoya $\left(10^{\circ} \mathrm{N} \& 85^{\circ} \mathrm{W}\right)$, Pacífico, Costa Rica, fueron hechas a bordo de los cruceros de los buques científicos Skimmer (Febrero y julio de 1979, abril de 1980) y V. Hensen (Diciembre de 1993 y febrero de 1994). Las descripciones de los métodos de captura y de la abundancia, diversidad y distribución de las especies, están incluídas en Bartels, Price, López y Bussing (1983, 1984) y en Wolff (1996), respectivamente. No obstante, aportamos aquí un resumen de los métodos: En el buque Skimmer las recolectas se hicieron en 20 estaciones y en un ámbito de profundidad de 10 a $50 \mathrm{~m}$ (Fig. 1A y Fig. 1C). Se utilizó una red de arrastre tipo otter (Fig. 2A) con malla de $3.6 \mathrm{~cm}$ en el cono final receptor de la captura. En el buque V. Hensen las capturas se hicieron en 12 estaciones entre 10 y $228 \mathrm{~m}$ de profundidad (Fig. 1B y Fig. 1D) y se utilizaron dos tipos de red: otter (malla 2.5 $\mathrm{cm})$ y beam (malla de $1 \mathrm{~cm}$ ).

Una vez que la red fue depositada y abierta sobre la cubierta de los buques se procedió a separar los peces en grupos similares por su aspecto externo (Fig. 2B). Los ejemplares de esos grupos fueron revisados por M. López \& W. Bussing (Fig. 2C) quienes realizaron, a bordo de ambos cruceros, la identificación de la mayoría de las especies y seleccionaron ejemplares testigo. Los ejemplares seleccionados fueron preservados en formalina (10\% en agua de mar), luego conservados en alcohol etílico (70\%), catalogados, e ingresados a la Colección Ictiológica (Angulo, López,
Bussing, Ramírez-Coghi, \& Arias-Godínez, 2015) del Museo de Zoología de la Universidad de Costa Rica. En ambos buques se hizo a bordo la medición de las longitudes totales de la mayoría de los peces identificados (Fig. 2D).

En este estudio se revisaron los datos de longitudes de peces publicados por Price, Bussing, Maurer y Bartels (1980) y por Wolff y Vargas (1994), para los buques Skimmer y V. Hensen, respectivamente. Además, se consultaron las listas originales de especies en Price et al. (1980) y en Wolff \& Vargas (1994). Se seleccionaron las cinco especies con las longitudes mayores.

Para actualizar las listas de especies de peces recolectados por ambos buques, se procedió a verificar los nombres citados en Bartels, Price, López y Bussing (1983) (Tabla 1: 189 taxa, 157 a nivel de especie) y en Bussing y López (1996) (Tabla 1: 211 taxa, 185 a nivel de especie), respectivamente. A la lista del Golfo de Nicoya se le agregaron las 20 especies incluidas por Bussing y López (1996) como pertenecientes a las estaciones externas (offshore) del Golfo (51, 52, 53, 54, Fig. 1B). Se procedió luego a revisar las listas digitalizadas comparándolas con los datos publicados por Bussing y López (1993), Bearez (1996), Castellanos-Galindo, Rubio-Rincón, Beltrán-León, Zapata, y Baldwin (2006), Bussing y López (2009, 2015), Angulo et al., (2015), Murase et al., (2014), Espinoza, Díaz, Angulo, Hernández y Clarke (2018) y Smith (2018). También se consultaron las bitácoras de W. B. y M. L., para ambos cruceros. Seguidamente, se revisaron los ejemplares provenientes de esos cruceros y depositados la Colección Ictiológica del Museo de Zoología de la Universidad de Costa Rica (MZUCR) y se agregaron los nombres de las especies en la Colección identificadas o descritas posteriormente a las publicaciones de Bartels et al., (1983) y Bussing y López (1996). Luego, los nombres científicos fueron verificados contra los aceptados actualmente, según la página electrónica World Register of Marine Species (WORMS) y se hicieron los cambios pertinentes. Las listas en Bartels et al., (1983) y Bussing y López (1996), incluyen los nombres 

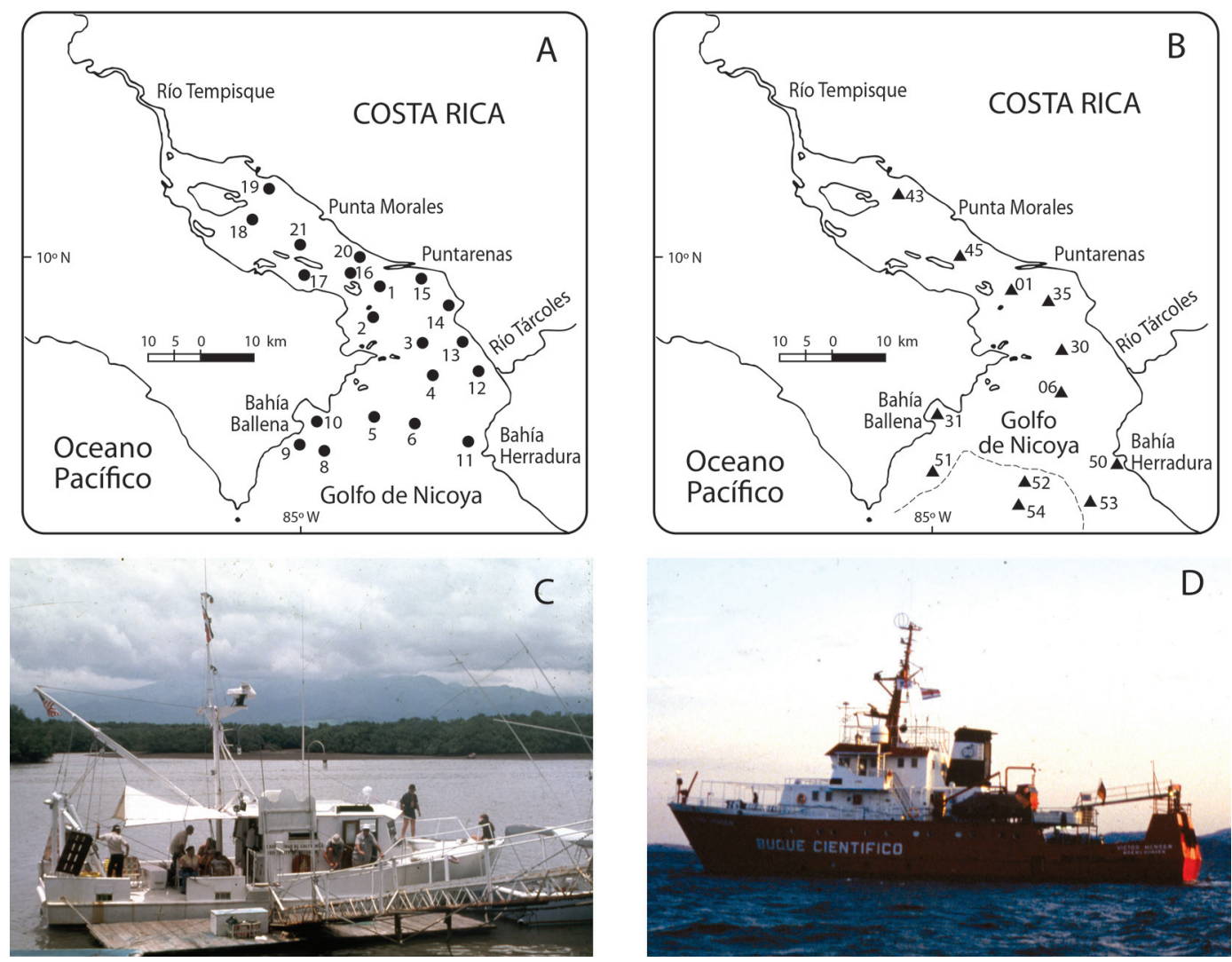

Fig. 1. Localización de las estaciones de recolecta de peces mediante redes epibénticas (Redes de arrastre tipo otter trawl y beam trawl) en el Golfo de Nicoya, Pacífico, Costa Rica: A, C. Buque Skimmer (Otter trawl; 20 estaciones) Febrero y julio de 1979, abril de 1980. Profundidad: 10 m a 50 m. Modificada de Fig. 1 (Bartels, Price, López, \& Bussing, 1983). B, D. Buque Víctor Hensen (Otter trawl y beam trawl, 12 estaciones) Diciembre 1993 y febrero 1994. Profundidad: $10 \mathrm{~m}$ a $228 \mathrm{~m}$. Modificada de Fig. 1 (Wolff, 1996). La línea discontínua indica la isóbata de $100 \mathrm{~m}$. Las profundidades de las estaciones a lo largo del eje central del Golfo, son: 43 (10 m), 45 (15 m), 01 (33 m), 30 (30 m), 06 (43 m), 52 (118 m), 54 (228 m). La amplitud promedio de la marea es de $3 \mathrm{~m}$. Fotos: K. Price y J. A. Vargas.

Fig. 1. Location of the fish collecting stations with epibenthic trawl nets (Otter and beam trawls) in the Gulf of Nicoya, Pacific, Costa Rica. A, C. Research vessel Skimmer (Otter trawl, 20 stations), February and July, 1979; April, 1980. Depth: 10 to 50 m. Modified from Fig. 1 (Bartels, Price, López \& Bussing, 1983). B, D. Reseach vessel Victor Hensen (Otter trawl and beam trawl, 12 stations). December 1993 and February 1994. Depth: 10 to 228 m. Modified from Fig. 1 (Wolff, 1996). The discontinuos line indicates the $100 \mathrm{~m}$ depth contour. The depths of the stations along the central axis of the Gulf, are: $43(10 \mathrm{~m}), 01(33 \mathrm{~m}), 30(30 \mathrm{~m}), 06(43 \mathrm{~m}), 52(118 \mathrm{~m}), 54(228 \mathrm{~m})$. The average tidal range is $3 \mathrm{~m}$. Photos: K. Price and J. A. Vargas.

agrupados en familias. En este estudio hemos optado, para facilitar su localización, el ordenar alfabéticamente los nombres de las especies y asignarles un código numérico.

Con el propósito de aportar información sobre la presencia relativa de algunas especies en las estaciones de cada crucero, se incluyeron los nombres de las 12 especies capturadas en un mayor número de estaciones, así como ejemplos de 10 especies presentes solamente en una estación.

Para proveer un marco de referencia sobre la variación de tres parámetros ambientales a lo largo de una gradiente de profundidad en 

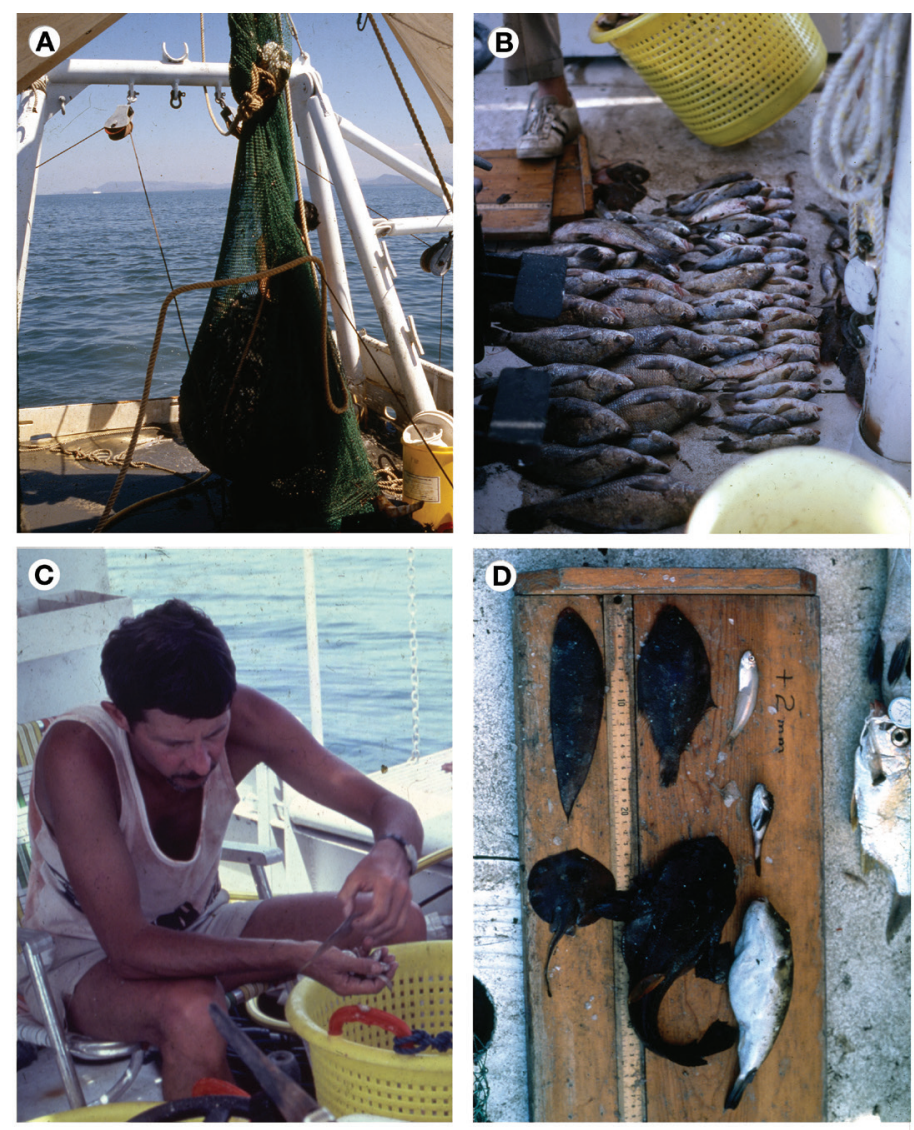

Fig. 2. A-E. Buque Skimmer. A. Red tipo otter en la cubierta después de un arrastre. B. Grupo de peces ordenado para su identificación y medición. C. W. Bussing († 2014) realiza a bordo la identificación de los peces. D. Soporte de madera, marcado en cm, para la medición de longitud total. Fotos: K. Price.

Fig. 2. A-E. Research vessel Skimmer. A. Otter trawl net hanging on deck after trawling. B. Group of fish organized for their indentification and measurement. C. W. Bussing $(\uparrow 2014)$ performing on board the species identifications. D. Wooden board marked in $\mathrm{cm}$, for the measurement of total lenghts. Photos: K. Price.

el Golfo de Nicoya, incluimos información con base en Wolff y Vargas (1994) sobre perfiles verticales (superficie-fondo), obtenidos mediante un sensor electrónico, de la salinidad, temperatura y oxigeno disuelto, en tres estaciones $(06,52$ y 54; Fig. 1B) del V. Hensen en el eje central del estuario.

\section{RESULTADOS}

La lista actualizada de las 250 especies de peces capturadas por los buques Skimmer y V. Hensen se incluye en la tabla 1. A las dos listas originales se les hizo un total de 41 actualizaciones a los nombres. Los nombres en las publicaciones originales aparecen en la columna izquierda de la tabla 1 y en la columna derecha los actualizados. Algunos ejemplares identificados provisionalmente a nivel de género en las listas originales correspondieron a formas juveniles y su asignación a una especie en particular es insegura basada en criterios morfológicos, por lo que no se incluyeron en la lista actualizada. Del total de especies con código numérico en la tabla 1, las recolectadas por el buque Skimmer fueron 157, mientras que 205 estuvieron representadas en los arrastres del V. Hensen. 
TABLA 1

Peces recolectados con redes de arrastre (Otter trawl y beam trawl) por los buques científicos Skimmer $(S, 1979-1980)^{1}$ y Victor Hensen $(H, 1993-1994)^{2}$, Golfo de Nicoya, Pacífico, Costa Rica

TABLE 1

Fishes collected with trawl nets (Otter trawl and beam trawl) by the research vessels Slimmer $(S, 1979-1980)^{1}$ and Victor Hensen $(H, 1993-1994)^{2}$, Gulf of Nicoya, Pacific, Costa Rica

\begin{tabular}{|c|c|c|c|}
\hline Código & Especie & Buques & Nombre actualizado \\
\hline 001 & Achirus klunzingeri & $H$ & A. klunzingeri (Steindachner, 1880) \\
\hline 002 & Achirus mazatlanus & $S, H$ & A. mazatlanus (Steindachner, 1869) \\
\hline 003 & Achirus scutum & $S, H$ & A. scutum (Günther, 1862) \\
\hline 004 & Albula nemoptera & $H$ & A. nemoptera (Fowler, 1911) \\
\hline 005 & Alectis ciliaris & $S$ & A. ciliaris (Bloch, 1787) \\
\hline 006 & Alphestes multiguttatus & $H$ & A. multiguttatus (Günther, 1867) \\
\hline 007 & Anchoa eigenmannia & $S$ & A. eigennmannia (Meek \& Hildebrand,1923) \\
\hline 008 & Anchoa ischana & $S, H$ & A. ischana (Jordan \& Gilbert, 1882) \\
\hline 009 & Anchoa lucida & $S, H$ & A. lucida (Jordan \& Gilbert, 1882) \\
\hline 010 & Anchoa nasus & $S, H$ & A. nasus (Kner \& Steindachner,1867) \\
\hline 011 & Anchoa panamensis & $S$ & A. panamensis (Steindachner, 1876) \\
\hline 012 & Anchoa sipinifer & $S$ & A. spinifer (Valenciennes, 1848) \\
\hline 013 & Anchoa starksi & $S, H$ & A. starksi (Gilbert \& Pierson, 1898) \\
\hline 014 & Anchoa walkeri & $S, H$ & A. walkeri Baldwin \& Chang, 1970 \\
\hline 015 & Anchovia macrolepidota & $S, H$ & A. macrolepidota (Kner, 1863) \\
\hline 016 & Ancylopsetta dendritica & $S, H$ & A. dendritica Gilbert, 1890 \\
\hline 017 & Anisostremus dovii & $S, H$ & Genyatremus dovii (Günther, 1864) \\
\hline 018 & Anisostremus pacifici & $S, H$ & Genyatremus pacifici (Günther, 1864) \\
\hline 019 & Antennarius avalonis & $S, H$ & Fowlerichthys avalonis (Jordan \& Starks, 1907) \\
\hline 020 & Apogon dovii & $S$ & A. dovii Günther, 1862 \\
\hline 021 & Argentina aliceae & $H$ & A. aliceae Cohen \& Atsaides, 1969 \\
\hline 022 & Arius dasycephalus & $S, H$ & Cathorops dasycephalus (Günther, 1864) \\
\hline 023 & Arius furthii & $S$ & Cathorops fuerthii (Steindachner, 1876) \\
\hline 024 & Arius jordani & $S$ & Ariopsis seemanni (Günther, 1864) \\
\hline 025 & Arius kessleri & $H$ & Notarius kessleri (Steindachner, 1876) \\
\hline 026 & Arius osculus & $H$ & Notarius osculus (Jordan \& Gilbert, 1883) \\
\hline \multirow[t]{2}{*}{027} & Arius platypogon & $H$ & Occidentarius platypogon (Günther, 1864) \\
\hline & Arius seemani & $S$ & Ariopsis seemanni (Günther, 1864) \\
\hline \multirow[t]{5}{*}{028} & Arius steindachneri & $S$ & Cathorops steindachneri (Gilbert \& Starks, 1904) \\
\hline & Arius sp. 1 & $S$ & Tabla 2 \\
\hline & Arius sp. A & $H$ & Tabla 2 \\
\hline & Arius sp. 2 & $S$ & Tabla 2 \\
\hline & Arius sp. B & $H$ & Tabla 2 \\
\hline \multirow[t]{2}{*}{029} & Arothron hispidus & $H$ & A. hispidus (Linnaeus, 1758) \\
\hline & Auxis sp. & $H$ & Tabla 2 \\
\hline 030 & Azevia panamensis & $S, H$ & Cyclopsetta panamensis (Steindachner, 1876) \\
\hline \multirow[t]{2}{*}{031} & Bairdiella armata & $S, H$ & B. armata Gill, 1863 \\
\hline & Bairdiella sp. & $S$ & Tabla 2 \\
\hline 032 & Batrachoides gilberti & $S$ & B. gilberti Meek \& Hildebrand, 1928 \\
\hline 033 & Batrachoides pacificum & $S$ & B. pacifici (Günther, 1861) \\
\hline 034 & Bellator gymnostethus & $S, H$ & B. gymnostethus (Gilbert, 1892) \\
\hline
\end{tabular}


TABLA 1 (Continuación) / TABLE 1 (Continued)

\begin{tabular}{|c|c|c|c|}
\hline Código & Especie & Buques & Nombre actualizado \\
\hline 035 & Bellator laxias & $H$ & B. loxias (Jordan, 1897) \\
\hline 036 & Bellator xenisma & $H$ & B. xenisma (Jordan \& Bolmann, 1890) \\
\hline 037 & Bollmannia chlamydes & $S, H$ & B. chlamydes Jordan 1890 \\
\hline \multirow[t]{2}{*}{038} & Bollmannia stigmatura & $S, H$ & B. stigmatura Gilbert, 1892 \\
\hline & Bollmannia sp. & $H$ & Tabla 2 \\
\hline 039 & Bregmaceros bathymaster & $H$ & B. bathymaster Jordan \& Bollman, 1890 \\
\hline 040 & Brotula clarkae & $S, H$ & B. clarkae Hubbs, 1944 \\
\hline 041 & Calamus brachysomus & $H$ & C. brachysomus (Lockington, 1880) \\
\hline 042 & Cathorops tuyra & $H$ & C. tuyra (Meek \& Hildebrand, 1923) \\
\hline 043 & Caranx caballus & $H$ & C. caballus Günther, 1868 \\
\hline 044 & Caranx caninus & $H$ & C. caninus Günther, 1867 \\
\hline 045 & Caranx otrynter & $H$ & Carangoides otrynter (Jordan \& Gilbert, 1883) \\
\hline 046 & Caranx speciosus & $H$ & Gnathanodon speciosus (Forsskal, 1775) \\
\hline 047 & Caranx vinctus & $S, H$ & C. vinctus Jordan \& Gilbert, 1882 \\
\hline \multirow[t]{2}{*}{048} & Caulolatilus affinis & $H$ & C. princeps (Jenyns, 1840) \\
\hline & Caulolatilus sp. & $S$ & Tabla 2 \\
\hline 049 & Centropomus armatus & $S$ & C. armatus Gill, 1863 \\
\hline 050 & Centropomus nigrescens & $S$ & C. nigrescens Günther, 1864 \\
\hline 051 & Cephalopholis acanthistius & $S$ & Hyporthodus acanthistius (Gilbert, 1892) \\
\hline 052 & Cetengraulis mysticetus & $S, H$ & C. mysticetus (Günther, 1867) \\
\hline 053 & Chaetodipterus zonatus & $S, H$ & C. zonatus (Girard, 1858) \\
\hline 054 & Chiloconger labiatus & $H$ & C. dentatus (Garman, 1899) \\
\hline \multirow[t]{2}{*}{055} & Chloroscombrus orqueta & $S$ & C. orqueta Jordan \& Gilbert, 1883 \\
\hline & Chloropthalmus sp. & $H$ & Tabla 2 \\
\hline 056 & Citharichthys gilberti & $S, H$ & C. gilberti Jenkins \& Evermann, 1889 \\
\hline 057 & Citharichthys platophrys & $S, H$ & C. platophrys Gilbert, 1891 \\
\hline 058 & Coeolorhinchus scaphopsis & $H$ & C. scaphopsis (Gilbert,1890) \\
\hline 059 & Coryphaeonoides leucophaeus & $H$ & C. capito (Garman, 1893) \\
\hline 060 & Cyclopsetta querna & $S, H$ & C. querna (Jordan \& Bollman, 1890) \\
\hline 061 & Cynoponticus coniceps & $S, H$ & C. coniceps (Jordan \& Gilbert, 1882) \\
\hline 062 & Cynoscion albus & $S, H$ & C. albus (Günther, 1864) \\
\hline 063 & Cynoscion phoxocephalus & $S, H$ & C. phoxocephalus Jordan \& Gilbert, 1882 \\
\hline 064 & Cynoscion reticulatus & $S, H$ & C. reticulatus (Günther, 1864) \\
\hline 065 & Cynoscion squamipinnis & $S, H$ & C. squamipinnis (Günther, 1867) \\
\hline 066 & Cynoscion stolzmanni & $S, H$ & C. stolzmanni (Steindachner, 1879) \\
\hline 067 & Dasyatis longus & $S, H$ & Hypanus longus (Garman, 1880) \\
\hline 068 & Decodon melasma & $H$ & D. melasma Gomon, 1974 \\
\hline 069 & Diapterus aureolus & $S, H$ & Deckertichthys aureolus (Jordan \& Gilbert, 1882) \\
\hline 070 & Diapterus peruvianus & $S, H$ & D. peruvianus (Cuvier, 1830) \\
\hline 071 & Diodon hystrix & $H$ & D. hystrix Linnaeus, 1758 \\
\hline 072 & Diplectrum eumelum & $H$ & D. eumelum Rosenblatt \& Johnson, 1974 \\
\hline 073 & Diplectrum euryplectrum & $H$ & D. euryplectrum Jordan \& Bollman, 1890 \\
\hline 074 & Diplectrum labarum & $S, H$ & D. labarum Rosenblatt \& Johnson, 1974 \\
\hline 075 & Diplectrum macropoma & $S, H$ & D. macropoma (Günther, 1864) \\
\hline 076 & Diplectrum maximum & $H$ & D. maximum Hildebrand, 1946 \\
\hline 077 & Diplectrum rostrum & $H$ & D. rostrum Bortone, 1974 \\
\hline 078 & Diplectrum pacificum & $S, H$ & D. pacificum Meek \& Hildebrand, 1925 \\
\hline
\end{tabular}


TABLA 1 (Continuación) / TABLE 1 (Continued)

\begin{tabular}{|c|c|c|c|}
\hline Código & Especie & Buques & Nombre actualizado \\
\hline 079 & Diplobatis ommata & $H$ & D. ommata (Jordan \& Gilbert, 1890) \\
\hline 080 & Elattarchus archidium & $S$ & E. archidium (Jordan \& Gilbert, 1882) \\
\hline \multirow[t]{2}{*}{081} & Engyophrys sanctilaurentii & $S, H$ & E. santilaurentii Jordan \& Bollman, 1890 \\
\hline & Epinephelus acanthistius & $H$ & Hyporthodus acanthistius (Gilbert, 1892) \\
\hline 082 & Epinephelus cifuentesi & $H$ & E. cifuentesi Lavenberg \& Grove, 1993 \\
\hline 083 & Epinephelus exul & $H$ & Hyporthodus exul (Fowler, 1944) \\
\hline 084 & Epinephelus niphobles & $H$ & Hyporthodus niphobles (Gilbert \& Starks, 1897) \\
\hline 085 & Epinephelus niveatus & $S$ & Hyporthodus niveatus (Valenciennes, 1828) \\
\hline 086 & Etropus crossotus & $S, H$ & E. crossotus Jordan \& Gilbert, 1882 \\
\hline 087 & Etropus peruvianus & $H$ & E. peruvianus Hildebrand, 1946 \\
\hline 088 & Eucinostomus argenteus & $S, H$ & E. argenteus Baird \& Girard, 1855 \\
\hline 089 & Eucinostomus currani & $H$ & E. currani Zahuranec, 1980 \\
\hline 090 & Eucinostomus gracilis & $S, H$ & E. gracilis (Gill, 1862) \\
\hline 091 & Gerres cinereus & $H$ & G. cinereus (Wabaum, 1792) \\
\hline 092 & Gobiesox milleri & $H$ & G. milleri Briggs, 1955 \\
\hline 093 & Gobioides peruvianus & $S, H$ & G. peruanus (Steindachner, 1880) \\
\hline 094 & Gobionellus liolepis & $S$ & G. liolepis (Meek \& Hildebrand, 1928) \\
\hline 095 & Gymnothorax dovii & $S$ & G. dovii (Günther, 1870) \\
\hline \multirow[t]{2}{*}{096} & Gymnothorax equatorialis & $S, H$ & G. equatorialis (Hildebrand,1946) \\
\hline & Gymnothorax sp. nov. & $H$ & Tabla 2 \\
\hline 097 & Haemulon scudderi & $H$ & H. scudderii Gill, 1862 \\
\hline 098 & Haemulopsis axilaris & $S$ & H. axilaris (Steindachner, 1869) \\
\hline 099 & Haemulopsis elongatus & $S, H$ & H. elongatus (Steindachner, 1879) \\
\hline 100 & Haemulopsis leuciscus & $S, H$ & H. leuciscus (Günther, 1864) \\
\hline 101 & Haemulopsis nitidus & $S, H$ & H. nitidus (Steindachner, 1869) \\
\hline 102 & Hemanthias peruanus & $S, H$ & H. peruanus (Steindachner, 1875) \\
\hline 103 & Hemanthias spinifer & $H$ & H. signifer (Garman, 1899) \\
\hline 104 & Hemicaranx leucurus & $S, H$ & H. leucurus (Günther, 1864) \\
\hline 105 & Hildebrandia nitens & $S, H$ & Rhynchoconger nitens (Jordan \& Bollman,1890) \\
\hline 106 & Himantura pacifica & $H$ & Styracura pacifica (Beebe \& Tee-Van, 1941) \\
\hline 107 & Hippocampus ingens & $S$ & H. ingens Girard, 1858 \\
\hline 108 & Hippoglossina bolmani & $H$ & H. bollmani Gilbert, 1890 \\
\hline 109 & Hippoglossina tetropthalmus & $S, H$ & H. tetrophthalma (Gilbert, 1890) \\
\hline 110 & Hoplopagrus guentheri & $S, H$ & H. guentherii Gill, 1862 \\
\hline 111 & Hoplunnis pacificus & $S$ & H. pacifica Lane \& Stewart, 1968 \\
\hline 112 & Ilisha furthii & $S, H$ & I. fuerthii (Steindachner, 1875) \\
\hline 113 & Isopisthus altipinnis & $S, H$ & I. remifer Jordan \& Gilbert, 1882 \\
\hline 114 & Kathetostoma averruncus & $H$ & K. averruncus Jordan \& Bollman, 1890 \\
\hline 115 & Kyphosus elegans & $S$ & K. elegans (Peters, 1869) \\
\hline 116 & Larimus acclivis & $S, H$ & L. acclivis Jordan \& Bristol, 1898 \\
\hline 117 & Larimus argenteus & $S$ & L. argenteus (Gill, 1863) \\
\hline 118 & Larimus effulgens & $S$ & L. effulgens Gilbert, 1898 \\
\hline 119 & Larimus pacificus & $S, H$ & L. pacificus Jordan \& Bollman, 1890 \\
\hline 120 & Lepophidium negropinna & $H$ & L. negropinna Hildebrand \& Barton, 1949 \\
\hline 121 & Lepophidium pardale & $S$ & L. pardale (Gilbert, 1890) \\
\hline 122 & Lepophidium prorates & $S, H$ & L. prorates (Jordan \& Bollman, 1890) \\
\hline 123 & Lycengraulis poeyi & $S$ & L. poeyi (Kner, 1863) \\
\hline
\end{tabular}


TABLA 1 (Continuación) / TABLE 1 (Continued)

\begin{tabular}{|c|c|c|c|}
\hline Código & Especie & Buques & Nombre actualizado \\
\hline & Lioglossina tetraophthalmus & S & Hippoglossina tetrophthalma (Gilbert, 1890) \\
\hline 124 & Lophiodes caulinaris & $S, H$ & L. caulinaris (Garman, 1899) \\
\hline 125 & Lophiodes setigerus & $S$ & Lohiomus setigerus (Vahl, 1797) \\
\hline 126 & Lophiodes spirulus & $H$ & L. spilurus (Garman,1899) \\
\hline 127 & Lutjanus argentiventris & $H$ & L. argentiventris (Peters, 1869) \\
\hline 128 & Lutjanus colorado & $S$ & L. colorado Jordan \& Gilbert, 1882 \\
\hline 129 & Lutjanus guttatus & $S, H$ & L. guttatus (Steindachner, 1869) \\
\hline 130 & Lutjanus peru & $S, H$ & L. peru (Nichols \& Murphy, 1922) \\
\hline 131 & Menticirrhus nasus & $S, H$ & M. nasus (Günther, 1868) \\
\hline 132 & Menticirrhus panamensis & $S, H$ & M. panamensis (Steindachner, 1876) \\
\hline 133 & Microgobius erectus & $S, H$ & M. erectus Ginsburg, 1938 \\
\hline 134 & Micropogonias altipinnis & $S$ & M. altipinnis (Günther, 1864) \\
\hline \multirow[t]{2}{*}{135} & Monolene maculipinna & $H$ & M. maculipinna Garman, 1899 \\
\hline & Muarenesox coniceps & $S$ & Cynoponticus coniceps (Jordan \& Gilbert, 1882) \\
\hline 136 & Mulloides dentatus & $H$ & Mulloidichthys dentatus (Gill, 1862) \\
\hline 137 & Mustelus lunulatus & $H$ & M. lunulatus Jordan \& Gilbert, 1882 \\
\hline 138 & Myrichthys tigrinus & $S, H$ & M. tigrinus Girard, 1859 \\
\hline 139 & Narcine brasiliensis & $H$ & N. brancroftii (Griffith \& Smith, 1834) \\
\hline 140 & Narcine entemedor & $S$ & N. entemedor Jordan \& Starks, 1895 \\
\hline 141 & Nebris occidentalis & $S, H$ & N. occidentalis Vaillant, 1897 \\
\hline 142 & Neobythites stelliferoides & $H$ & N. stelliferoides Gilbert, 1890 \\
\hline 143 & Neoopisthopterus tropicus & $S, H$ & N. tropicus (Hildebrand, 1946) \\
\hline 144 & Netuma platypogon & $S$ & Occidentarius platypogon (Günther, 1864) \\
\hline \multirow[t]{4}{*}{145} & Ophichthus remiger & $H$ & O. remiger (Valenciennes, 1847) \\
\hline & Ophichthus sp. B & $H$ & Tabla 2 \\
\hline & Ophichthus sp. D & $H$ & Tabla 2 \\
\hline & Ophidion sp. & $S$ & Tabla 2 \\
\hline 146 & Ophioscion imiceps & $S$ & O. imiceps (Jordan \& Gilbert, 1882) \\
\hline 147 & Ophioscion sciera & $S, H$ & O. scierus (Jordan \& Gilbert, 1884) \\
\hline 148 & Ophioscion typicus & $S, H$ & O. typicus Gill, 1863 \\
\hline 149 & Ophisoma prorigerum & $H$ & Ariosoma prorigerum (Gilbert, 1891) \\
\hline 150 & Opisthonema bulleri & $S$ & O. bulleri (Regan, 1904$)$ \\
\hline \multirow[t]{2}{*}{151} & Opisthonema libertate & $S$ & O. libertate (Günther, 1867) \\
\hline & Opisthonema sp. & $\mathrm{S}$ & Tabla 2 \\
\hline 152 & Opisthopterus dovii & $H$ & O. dovii (Günther, 1868) \\
\hline 153 & Opisthopterus equatorialis & $S, H$ & O. equatorialis Hildebrand, 1946 \\
\hline 154 & Orthopristis chalceus & $S$ & O. chalceus (Günther, 1864) \\
\hline 155 & Paralabrax humeralis & $S$ & P. humeralis (Valenciennes, 1828) \\
\hline 156 & Paralabrax loro & $H$ & P. loro Walford, 1936 \\
\hline 157 & Paralichthys woolmani & $H$ & P. woolmani Jordan \& Williams, 1897 \\
\hline 158 & Paralonchurus dumerilii & $S, H$ & P. dumerilii (Bocourt, 1869) \\
\hline 159 & Paralonchurus rathbuni & $H$ & P. rathbuni (Jordan \& Bollman, 1890) \\
\hline 160 & Parapsettus panamensis & $S, H$ & P. panamensis (Steindachner, 1876) \\
\hline 161 & Peprilus medius & $S, H$ & P. medius (Peters, 1869) \\
\hline 162 & Peprilus snyderi & $S, H$ & P. snyderi (Gilbert \& Starks, 1904) \\
\hline 163 & Perissias taeniopterus & $H$ & P. taeniopterus (Gilbert, 1890) \\
\hline 164 & Peristedion barbiger & $H$ & P. barbiger Garman, 1899 \\
\hline
\end{tabular}


TABLA 1 (Continuación) / TABLE 1 (Continued)

\begin{tabular}{|c|c|c|c|}
\hline Código & Especie & Buques & Nombre actualizado \\
\hline 165 & Peristedion crustosum & $H$ & P. crustosum Garman, 1899 \\
\hline 166 & Physiculus nematopus & $S, H$ & P. nematopus Gilbert, 1890 \\
\hline 167 & Physiculus rastrelliger & $H$ & P. rastrelliger Gilbert, 1890 \\
\hline 168 & Pliosteostoma lutipinnis & $H$ & P. lutipinnis (Jordan \&Gilbert, 1892) \\
\hline 169 & Polydactylus approximans & $S, H$ & P. approximans (Lay \& Bennett, 1839) \\
\hline 170 & Polydactylus opercularis & $S$ & Filimanus sealei (Jordan \&Richardson, 1910) \\
\hline 171 & Pomadasys branickii & $H$ & P. branickii (Steindachner, 1879) \\
\hline 172 & Pomadasys macracanthus & $S, H$ & P. macracanthus (Günther, 1864) \\
\hline 173 & Pomadasys panamensis & $S$ & P. panamensis (Steindachner, 1876) \\
\hline 174 & Pontinus furcirhinus & $H$ & P. furcirhinus Garman, 1899 \\
\hline \multirow[t]{2}{*}{175} & Pontinus sierra & $H$ & P. sierra (Gilbert, 1890) \\
\hline & Pontinus sp. nov. & $H$ & Tabla 2 \\
\hline 176 & Porichthys greenei & $H$ & P. greenei Gilbert \& Starks, 1904 \\
\hline \multirow{3}{*}{177} & Porichthys margaritatus & $S, H$ & P. margaritatus (Richardson, 1844) \\
\hline & Porichthys nautopaedium & $H$ & P. margaritatus (Richardson, 1844) \\
\hline & Priodonophus equatorialis & $S$ & Gymnothorax equatorialis (Hildebrand, 1946) \\
\hline \multirow[t]{2}{*}{178} & Prionotus albirostris & $S, H$ & P. albirostris Jordan \& Bollman, 1890 \\
\hline & Prionotus gimnostethus & $S$ & Bellator gymnostethus (Gilbert, 1892) \\
\hline 179 & Prionotus horrens & $S, H$ & P. horrens Richardson, 1844 \\
\hline 180 & Prionotus ruscarius & $S, H$ & P. ruscarius Gilbert \& Starks, 1904 \\
\hline 181 & Prionotus stephanophrys & $S, H$ & P. stephanophrys Lockingnton, 1881 \\
\hline 182 & Prionotus teaguei & $H$ & P. teaguei Briggs, 1956 \\
\hline 183 & Pristigenys serrula & $H$ & P. serrula (Gilbert, 1891) \\
\hline 184 & Pronotogrammus eos & $S, H$ & P. eos Gilbert, 1890 \\
\hline 185 & Pseudomyrophis micropinna & $H$ & P. micropinna Wade, 1946 \\
\hline \multirow[t]{2}{*}{186} & Pseudopeneus grandisquamis & $S, H$ & P. grandisquamis (Gill, 1863) \\
\hline & Pseudorhombus dendritica & $S$ & Ancylopsetta dendritica Gilbert, 1890 \\
\hline 187 & Pythonichthys asodes & $H$ & P. asodes Rosemblatt \& Rubinoff, 1972 \\
\hline 188 & Raja equatorialis & $S, H$ & R. equatorialis Jordan \& Bollman, 1890 \\
\hline 189 & Raja velezi & $S, H$ & R. velezi Chirichigno F., 1973 \\
\hline 190 & Rhinobatos leucorhyncus & $H$ & Pseudobatos leucorhynchus (Günther, 1867) \\
\hline 191 & Rypticus nigripinnis & $S, H$ & R. nigripinnis Gill, 1861 \\
\hline 192 & Sciadeichthys dowii & $H$ & Sciades dowii (Gill, 1863) \\
\hline 193 & Sciadeichthys troschelii & $S, H$ & Notarius troschelii (Gill, 1863) \\
\hline 194 & Scomber japonicus & $H$ & S. japonicus Houttuyn, 1782 \\
\hline 195 & Scorpaena histrio & $H$ & S. histrio Jenyns, 1840 \\
\hline 196 & Scorpaena mystes & $H$ & S. mystes Jordan \& Starks, 1895 \\
\hline 197 & Scorpaena russula & $S, H$ & S. russula_Jordan \& Bollman, 1890 \\
\hline \multirow[t]{2}{*}{198} & Selar crumenophthalmus & $H$ & S. crumenophthalmus (Bloch, 1793) \\
\hline & Selenaspis dowii & $H$ & Sciades dowii (Gill, 1863) \\
\hline 199 & Selene brevoortii & $S, H$ & S. brevoorti (Gill, 1863) \\
\hline 200 & Selene oerstedii & $S, H$ & S. oerstedii Lütken, 1880 \\
\hline 201 & Selene peruviana & $S, H$ & S. peruviana (Guichenot, 1866) \\
\hline 202 & Serranus aequidens & $H$ & S. aequidens Gilbert, 1890 \\
\hline 203 & Serranus psittacinus & $H$ & S. psittacinus Valenciennes, 1846 \\
\hline 204 & Sphoeroides annulatus & $S, H$ & S. annulatus (Jenyns, 1842) \\
\hline 205 & Sphoeroides fuerthii & $S, H$ & S. trichocephalus (Cope, 1870) \\
\hline
\end{tabular}


TABLA 1 (Continuación) / TABLE 1 (Continued)

\begin{tabular}{|c|c|c|c|}
\hline Código & Especie & Buques & Nombre actualizado \\
\hline 206 & Sphoeroides kendalli & S & S. kendalli Meek \& Hildebrand, 1928 \\
\hline 207 & Sphoeroides lobatus & $S, H$ & S. lobatus (Steindachner, 1870) \\
\hline \multirow[t]{2}{*}{208} & Sphoeroides sechurae & $S$ & S. sechrae Hildebrand, 1946 \\
\hline & Sphoeroides sp. 1 & $S$ & Tabla 2 \\
\hline 209 & Sphyraena ensis & $S$ & S. ensis Jordan \& Gilbert, 1882 \\
\hline 210 & Stellifer chrysoleuca & $S, H$ & S. chrysoleuca (Günter, 1867) \\
\hline 211 & Stellifer ericymba & $S$ & S. ericymba (Jordan \& Gilbert, 1882) \\
\hline 212 & Stellifer furthii & $S, H$ & S. fuerthii (Steindachner, 1876) \\
\hline 213 & Stellifer illecebrosus & $S, H$ & S. illecebrosus Gilbert, 1898 \\
\hline 214 & Stellifer mancorensis & $S, H$ & S. mancorensis Chirichigno F., 1962 \\
\hline 215 & Stellifer oscitans & $S, H$ & S. oscitans (Jordan \& Gilbert, 1882) \\
\hline \multirow[t]{2}{*}{216} & Stellifer zestocarius & $S, H$ & S. zestocarus Gilbert, 1898 \\
\hline & Stellifer $\mathrm{sp}$. & $S$ & Tabla 2 \\
\hline 217 & Syacium cf longidorsale & $H$ & S. cf longidorsale Murakami \& Amaoka, 1992 \\
\hline 218 & Syacium latifrons & $S, H$ & S. latifrons (Jordan \& Gilbert, 1882) \\
\hline 219 & Syacium ovale & $S, H$ & S. ovale (Günter, 1864) \\
\hline 220 & Symphurus atramentatus & $S, H$ & S. atramentatus Jordan \& Bollman, 1890 \\
\hline 221 & Symphurus callopterus & $H$ & S. callopterus Munroe \& Mahadeva, 1989 \\
\hline 222 & Symphurus chabanaudi & $H$ & S. chabanaudi Mahadeva \& Munroe, 1990 \\
\hline 223 & Symphurus elongatus & $H$ & S. elongatus (Günter, 1868) \\
\hline 224 & Symphurus fasciolarius & $S$ & S. fasciolaris Gilbert, 1892 \\
\hline 225 & Symphurus gorgonae & $H$ & S. gorgonae Chabanaud, 1948 \\
\hline 226 & Symphurus leei & $H$ & S. leei Jordan \& Bollman, 1890 \\
\hline 227 & Symphurus melanurus & $S, H$ & S. melanurus Clark, 1936 \\
\hline 228 & Symphurus oligomerus & $H$ & S. oligomerus Mahadeva \& Munroe, 1990 \\
\hline 229 & Symphurus undecimplerus & $H$ & S. undecimplerus Munroe \& Nizinski, 1990 \\
\hline 230 & Symphurus williamsi & $H$ & S. williamsi Jordan \& Cuvier, 1895 \\
\hline 231 & Synchiropus atrilabiatus & $H$ & S. atrilabiatus (Garman, 1899) \\
\hline 232 & Synodus evermanni & $S, H$ & S. evermanni Jordan \& Bolman, 1890 \\
\hline 233 & Synodus scituliceps & $S, H$ & S. scituliceps Jordan \& Gilbert, 1882 \\
\hline 234 & Synodus sechurae & $H$ & S. sechurae Hildebrand, 1946 \\
\hline 235 & Torpedo tremens & $H$ & Tetronarce tremens (de Buen, 1959) \\
\hline 236 & Trachinotus paitensis & $H$ & T. paitensis Cuvier, 1832 \\
\hline 237 & Trichiurus nitens & $S, H$ & T. lepturus Linneaus, 1758 \\
\hline 238 & Trinectes fimbriatus & $S$ & T. fimbriatus (Günter, 1862) \\
\hline \multirow[t]{3}{*}{239} & Trinectes fonsecensis & $S, H$ & T. fonsecensis (Günter, 1862) \\
\hline & Trinectes sp. & $S$ & Tabla 2 \\
\hline & Trinectes sp. nov & $H$ & Tabla 2 \\
\hline 240 & Umbrina bussingi & $H$ & U. bussingi López S.,1980 \\
\hline 241 & Umbrina xanti & $H$ & U. xanti Gill, 1862 \\
\hline 242 & Urolophus halleri & $S$ & U. halleri Cooper, 1863 \\
\hline 243 & Urotrygon aspidura & $S$ & U. aspidura (Jordan \& Gilbert, 1882) \\
\hline 244 & Urotrygon chilensis & $S, H$ & U. chilensis (Günter, 1872) \\
\hline 245 & Urotrygon munda & $S, H$ & U. munda Gill, 1863 \\
\hline 246 & Urotrygon rogersi & $H$ & U. rogersi (Jordan \& Starks, 1895) \\
\hline 247 & Urorygon nana & $H$ & U. nana Miyake \&McEachran, 1988 \\
\hline 248 & Urotrygon serrula & $S$ & U. serrula Hildebrand, 1946 \\
\hline
\end{tabular}


TABLA 1 (Continuación) / TABLE 1 (Continued)

\begin{tabular}{llll} 
Código & \multicolumn{1}{c}{ Especie } & Buques & \multicolumn{1}{c}{ Nombre actualizado } \\
249 & Urotrygon sp. nov & S & Tabla 2 \\
250 & Zalieutes elater & Zapteryx exasperata $H$ & Z. elater (Jordan \& Gilbert, 1882) \\
\hline
\end{tabular}

Columna izquierda: código, nombre en la publicación original. Columna central: Buques Skimmer $(\boldsymbol{S})^{1}$ y Hensen $(\boldsymbol{H})^{2}$. Columna derecha: nombre actualizado y autoridad.

1. Bartels, Price, López \& Bussing (1983): Cuadro 1 (Págs. 80-85).

2. Bussing \& López (1996): Cuadro 1 (Págs. 184-186).

Los cinco ejemplares de mayor longitud medidos en el buque Skimmer, fueron: el congrio Cynoponticus coniceps (103 cm. Código 061), la raya bruja Raya velezi $(65 \mathrm{~cm}$ de diámetro del disco. 189), el congrio Gymnothorax dovii $(59 \mathrm{~cm}, 095)$ y la corvina agria Micropogonias altipinnis $(57 \mathrm{~cm}, 134)$. Los cinco ejemplares de mayor longitud capturados por el buque V. Hensen, fueron: la raya látigo Hypanus longus (117 cm de diámetro. 067), el bagre Sciades dowii $(87 \mathrm{~cm} .192)$, la raya Narcine brancroftii (80 cm de diámetro. 139), el bonito Caranx caballus $(75 \mathrm{~cm} .043)$ y la corvina reina Cynoscion albus $(75 \mathrm{~cm} .062)$.

En la tabla 2 incluimos los nombres de las 18 especies adicionales producto de la revisión de la Colección Ictiológica del Museo de Zoología (MZUCR), por lo que el total de especies para ambos buques en el Golfo de Nicoya y entre 10 y $228 \mathrm{~m}$ de profundidad asciende a 268, más una especie de Pontinus en proceso de descripción de las estaciones 52 y 53. Ocho especies fueron descritas posteriormente a la publicación (1996) de la lista del V. Hensen: Gymnothorax phalarus, Notarius biffi, N. cookei, Ophictus mecopterus, O. melope, Sphoeroides rosenblatti, Trinectes xanthurus y Urotrygon cimar.

Las redes utilizadas capturaron preferentemente especies demersales, frecuentes sobre los sedimentos del estuario. No obstante, durante el tiempo en el cual la red es bajada al fondo o subida abierta desde este, se dio la captura de algunas especies presentes en la columna de agua. Estas especies de hábitos pelágicos también fueron incluidas en la lista.
De las 268 especies un total de 249 (93\%) correspondió a peces óseos (Osteichthyes). Los peces cartilaginosos (Chondrichthyes, 19 especies, $7 \%$ ) estuvieron representados por una especie de tiburón (Mustelus lunulatus), el pez diablo Zapteryx exasperata, el pez guitarra Pseudobatos leucorhyncus, las rayas eléctricas Diplobatis onmata, Narcine bancroftii, $N$. entemedor y Tetronarce tremens, y otras 12 rayas: Hypanus longus, Raja equatorialis, $R$. velezi, Styracura pacifica, Urolophus halleri, Urotrygon aspidura, U. cimar, U. chilensis, $U$. munda, U. nana, $U$. rogersi y $U$. serrula.

De las especies incluidas en la tabla $1 \mathrm{y}$ tabla 2, un total de 31 (11\%) son de la familia Sciaenidae (corvinas). Entre los géneros de corvinas capturados por ambos buques, están: Bairdiella, Cynoscion, Elattarchus, Isopisthus, Larimus, Menticirrhus, Micropogonias, Nebris, Ophioscion, Paralonchurus, Stellifer y Umbrina. El género más diverso fue Stellifer, con ocho especies.

Los peces conocidos como lenguados y peces hoja (Bothidae, Cynoglossidae, Paralichthyidae y Achiridae) habitantes propios del fondo del estuario, estuvieron representados por $32(12 \%)$ especies (Tabla 1 y Tabla 2) y 13 géneros (Achirus, Ancylopsetta, Citharichthys, Cyclopsetta, Engyophrys, Etropus, Hypoglossina, Monolene, Paralichthys, Perissias, Syacium, Symphurus y Trinectes). El género más diverso en los arrastres fue Symphurus, con 11 especies.

De la familia Serranidae (cabrillas y meros) fueron capturadas 21 especies (8 $\%$ ) de siete géneros (Alphestes, Diplectrum, 
TABLA 2

Otras especies de peces recolectadas en el Golfo de Nicoya en las expediciones de los buques científicos Skimmer y V. Hensen y catalogadas en la Colección de Ictiología del Museo de Zoología de la Universidad de Costa Rica (MZUCR), e identificadas o descritas después de las expediciones

TABLE 2

Other species of fish collected in the Gulf of Nicoya by the expeditions of the scientific ships Skimmer and V. Hensen and catalogued in the Ichthyology Collection of the Musem of Zoology, University of Costa Rica (MZUCR), and identified or described after the expeditions

\begin{tabular}{llcc} 
Código & \multicolumn{1}{c}{ Especie } & Localidad & Catálogo Museo UCR \\
251 & Auxis thazard (Lacepede, 1800) & Estación 53 Golfo & MZUCR 0878001 \\
252 & Bairdiella ensifera (Jordan \& Gilbert,1882) & Región superior Golfo & MZUCR 1526001 \\
253 & Bollmannia marginalis Ginsburg, 1939 & Estación 50 Golfo & MZUCR 2377010 \\
254 & Caulolatilus princeps (Jenyns, 1840) & Frente a Isla Tortuga & MZUCR 1209004 \\
255 & Chloropthalmus mento Garman, 1899 & Estación 54 Golfo & MZUCR 2777001 \\
256 & Gymnothorax phalarus Bussing, 1998 & Estación 51 Golfo & MZUCR 2373012 Holotipo \\
257 & Notarius osculus (Jordan \& Gilbert, 1883) & Frente a Manzanillo & MZUCR 1290004 \\
258 & Notarius biffi Betancur R., \& Acero P., 2004 & Frente a Manzanillo & MZUCR 2386015 \\
259 & Notarius cookei Acero P., \& Betancur R., 2002 & S. Pta. Gavilanes, I. Chira & MZUCR 1518002 \\
260 & Ophicthus frontalis Garman, 1899 & Boca del Golfo & MZUCR 2354002 \\
261 & Ophicthus mecopterus McCosker \& Rosenblatt, 1998 & Centro del Golfo & MZUCR 2352001 Paratipo \\
262 & Ophicthus melope McCosker \& Rosenblatt, 1998 & Estación 54 Golfo & MZUCR 2350002 Holotipo \\
263 & Ophidion scrippsae (Hubbs, 1916) & Frente a Bahia Ballena & MZUCR 1281009 \\
264 & Opistonema medirastre (Berry \&Barrett, 1963) & Frente a La Angostura & MZUCR 1510008 \\
265 & Sphoeroides rosenblatti Bussing, 1996 & Punta Morales & MZUCR 127013 Paratipo \\
266 & Stellifer ephelis Chirichigno F., 1974 & S.E. Isla Chira & MZUCR 1289001 \\
267 & Trinectes xanthurus Walker \& Bollinger, 2001 & N. Isla San Lucas & MZUCR 1513002 \\
268 & Urotrygon cimar López S. \& Bussing, 1998 & Frente a Manzanillo & MZUCR 2386001 Paratipo \\
& Pontinus sp. nov. & Estaciones 52, 53 Golfo & MZUCR 0626001 \\
\hline
\end{tabular}

Epinephelus, Hemanthias, Hyporthodus, Paralabrax, Pronotogrammus y Serranus). Las anchoas (Engraulidae) incluyeron 11 especies de los géneros Anchoa, Anchovia, Cetengraulis y Lycengraulis. Del grupo de los bagres o cuminates (Ariidae) fueron capturadas 10 especies de cinco géneros (Ariopsis, Cathorops, Notarius, Occidentarius y Sciades). Los roncadores (Haemulidae) aportaron 10 especies, de cuatro géneros (Anisostremus, Haemulon, Haemulopsis y Pomadasys). Los cabros (Triglidae), habitantes del fondo, aportaron ocho especies y dos géneros (Bellator y Prionotus). Las anguilas (Ophichthyidae), también habitantes de ambientes sedimentarios, estuvieron representadas por seis especies de los géneros Ophicthus, Pseudomyriophys y Myrichthys. Tres de las especies nuevas descritas pertenecen al género
Ophicthus (Tabla 2). Los pargos (Lutjanidae) estuvieron representados por cinco especies y dos géneros (Hoplopagrus y Lutjanus).

En la tabla 3 se anotaron ejemplos de 12 especies frecuentes en las 20 estaciones del buque Skimmer. Siete especies: Prionotus horrens (16 estaciones) Sphoeroides trichocephalus (14), Syacium ovale (13) Synodus scituliceps (13), Neoopisthopterus tropicus (12), Cyclopsetta querna (11) y Porichthys margaritatus (11) fueron capturadas en más del $50 \%$ de las estaciones.

En la tabla 4 se incluyeron ejemplos de 12 especies frecuentes en las 12 estaciones del buque V Hensen. Cinco especies: P. margaritatus (9 estaciones), Synodus scituliceps (8), Bollmannia chlamydes (6), Symphurus callopterus (6), S. elongatus (6) y Synodus evermanni (6) 
TABLA 3

Buque científico Skimmer: Ejemplos de 12 especies de peces (de un total de 157) más frecuentes en las 20 estaciones y códigos de las estaciones donde se les encontró. B. Ejemplos de 10 especies encontradas en una estación. Febrero y julio, 1979. Golfo de Nicoya, Pacífico, Costa Rica*

TABLE 3

Research vessel Skimmer: Examples of 12 species of fish (from a total of 157) more frequent in the 20 stations and station codes where found. B. Examples of 10 species found in one station. February and July, 1979.

Gulf of Nicoya, Pacífic, Costa Rica*

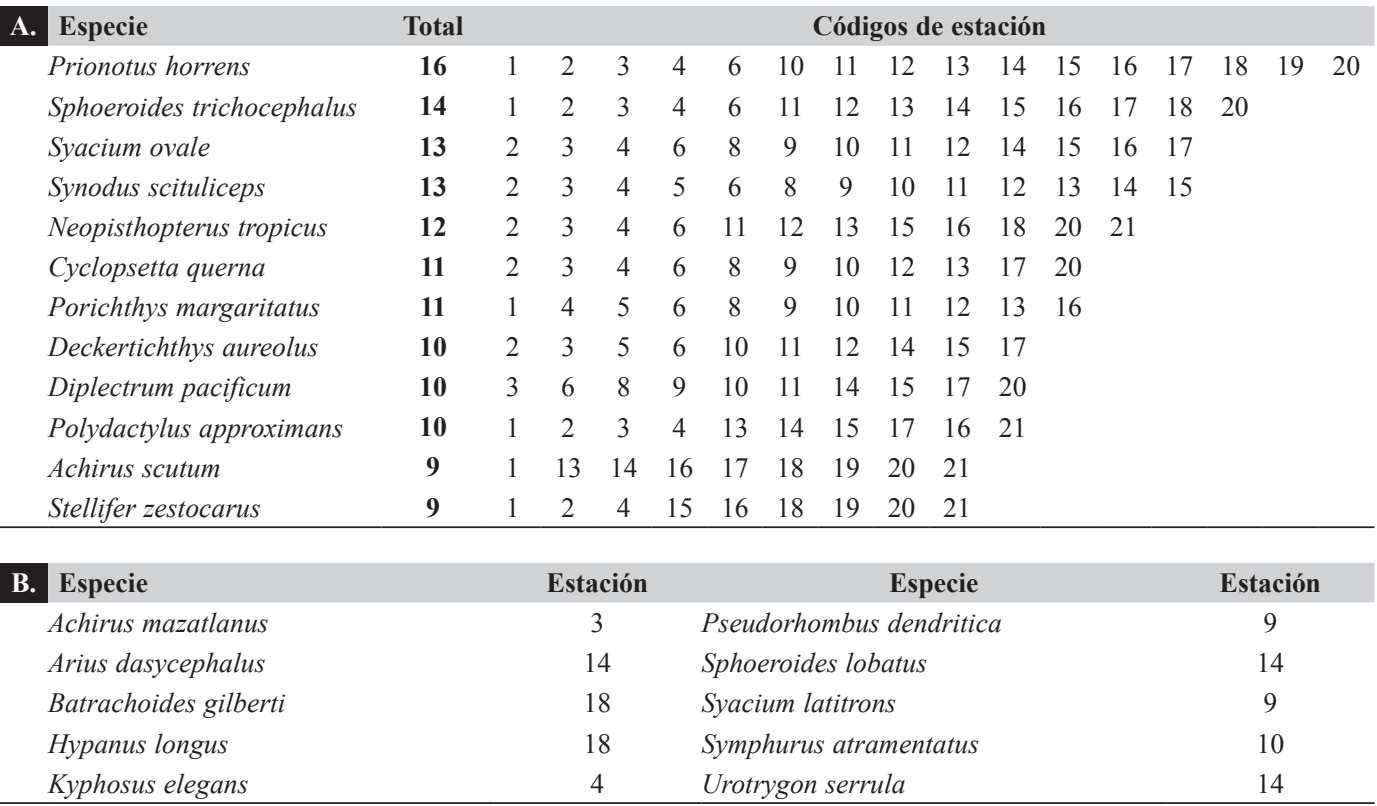

* Price, Bussing, Bussing, Maurer \& Bartels (1980): Cuadros I, II, IV,V (Pgs. 87-104).

fueron capturadas en el $50 \%$ de las estaciones. En la tabla 3 y tabla 4 también se citaron ejemplos de 10 especies encontradas en una sola estación por cada buque, respectivamente.

En la Fig. 3 se incluyeron dibujos de las doce especies más frecuentes en ambos cruceros.

En la Fig. 4 se dibujaron perfiles (superficie - fondo) de salinidad, temperatura y oxígeno disuelto en tres estaciones $(06,52,54)$ del V. Hensen en el eje central del Golfo de Nicoya. En esta región del estuario la salinidad varió entre $28 \%$ en la superficie y cerca de $35 \%$ en el fondo. La temperatura varió entre $28^{\circ} \mathrm{C}$ en la superficie, $20^{\circ} \mathrm{C}$ a los $60 \mathrm{~m}$ y 12 ${ }^{\circ} \mathrm{C}$ cerca de los $230 \mathrm{~m}$. En las estaciones $52 \mathrm{y}$ 54 se observó una termoclina entre los 40 y
$60 \mathrm{~m}$ (Fig. 4). La variación vertical del oxígeno disuelto (\% saturación) fue desde $100 \%$ cerca la superficie, $60 \%$ cerca de los $50 \mathrm{~m}$, y entre 5 y $2 \%$ a profundidades mayores de $150 \mathrm{~m}$. En la estación 06 se observó, a cinco metros bajo la superficie, una saturación mayor al $100 \%$.

\section{DISCUSIÓN}

El total de especies de peces presentes en la costa del Pacífico de Costa Rica fue estimado en 838 por Bussing y López (2009) hace más de una década. De ese total 719 correspondían a registros, entre la zona de entremareas y $200 \mathrm{~m}$ de profundidad, y depositados 
TABLA 4

Buque científico V. Hensen: Ejemplos de 12 especies de peces (de un total de 207) más frecuentes en las 12 estaciones y códigos de las estaciones donde se les encontró. B. Ejemplos de 10 especies encontradas en una estación.

Diciembre 1993 y Febrero 1994. Golfo de Nicoya, Pacífico, Costa Rica*

TABLE 4

Research vessel V. Hensen: Examples of 12 species of fish (from a total of 207) more frequent in the 12 stations and station codes where found. B. Examples of 10 species found in one station. December 1993 and February 1994. Gulf of Nicoya, Pacific, Costa Rica*

\begin{tabular}{|c|c|c|c|c|c|c|c|c|c|c|}
\hline A. Especie & Total & \multicolumn{9}{|c|}{ Códigos de estación } \\
\hline Porichthys margaritatus & 9 & 06 & 30 & 31 & 35 & 50 & 51 & 52 & 53 & 54 \\
\hline Synodus scituliceps & 8 & 01 & 06 & 30 & 31 & 35 & 50 & 52 & 53 & \\
\hline Bollmannia chlamydes & 6 & 06 & 31 & 50 & 52 & 53 & 54 & & & \\
\hline Symphurus callopterus & 6 & 06 & 31 & 50 & 52 & 53 & 54 & & & \\
\hline Synodus evermanni & 6 & 06 & 31 & 50 & 51 & 52 & 53 & & & \\
\hline Citharychthys platophrys & 5 & 01 & 06 & 52 & 53 & 54 & & & & \\
\hline Cyclopsetta querna & 5 & 01 & 06 & 30 & 35 & 45 & & & & \\
\hline Pontinus sierra & 5 & 31 & 51 & 52 & 53 & 54 & & & & \\
\hline Porichthys greenei & 5 & 01 & 06 & 30 & 35 & 45 & & & & \\
\hline Prionotus horrens & 5 & 01 & 06 & 30 & 31 & 45 & & & & \\
\hline Synchiropus attrilabiatus & 5 & 06 & 51 & 52 & 53 & 54 & & & & \\
\hline Zalieutes elater & 5 & 06 & 31 & 51 & 52 & 53 & & & & \\
\hline B. Especie & & \multicolumn{2}{|c|}{ Estación } & \multicolumn{4}{|c|}{ Especie } & & \multicolumn{2}{|c|}{ Estación } \\
\hline Arius dasycephalus & & \multicolumn{2}{|c|}{45} & \multicolumn{4}{|c|}{ Perissias taeniopterus } & & \multicolumn{2}{|c|}{51} \\
\hline Cathorops tuyra & & \multicolumn{2}{|c|}{43} & \multicolumn{4}{|c|}{ Menticirrhus panamensis } & & \multicolumn{2}{|c|}{01} \\
\hline Coeolorhynchus scaphopsis & & \multicolumn{2}{|c|}{54} & \multicolumn{4}{|c|}{ Monolene maculipina } & & \multicolumn{2}{|c|}{52} \\
\hline Diplectrum maximum & & \multicolumn{2}{|c|}{53} & \multicolumn{4}{|c|}{ Ophichthus remiger } & & \multicolumn{2}{|c|}{31} \\
\hline Gerres cinereus & & \multicolumn{2}{|c|}{31} & \multicolumn{4}{|c|}{ Urotrygon munda } & & \multicolumn{2}{|c|}{45} \\
\hline
\end{tabular}

*Wolff \& Vargas (1994): Apéndices I, II (Pgs. 84-92); Wolff (1996): Cuadro 1 (Pág. 180).

en la Colección del Museo de Zoología de la Universidad de Costa Rica (MZUCR). Esas 838 especies para Costa Rica indican una alta diversidad íctica, cuando se le compara, por ejemplo, con las 619 especies encontradas hasta $200 \mathrm{~m}$ de profundidad en la más extensa costa del Ecuador continental (Bearez, 1996). Las evaluaciones de la diversidad de la fauna ictiológica del Golfo de Nicoya con base en los arrastres hechos por los buques Skimmer y Victor Hensen datan de hace 40 y 25 años, respectivamente. Esos estudios fueron precedidos, entre otros, por el de Erdman (1971) quien, entre 1951 y 1953, hizo observaciones sobre 54 especies de peces capturadas con anzuelos y otras artes en la región interna del Golfo, y por el de León (1973) en el que mediante redes de arrastre se recolectaron 145 especies en 47 estaciones, desde la boca del Río Tempisque hasta la región media del estuario a la altura del puerto de Puntarenas.

Las 268 especies citadas en este estudio para el Golfo de Nicoya representan cerca del $32 \%$ de las 838 citadas por Bussing y López (2009) para la costa del Pacífico de Costa Rica. Recientemente, y con base en la Colección del Museo de Zoología (MZUCR), Murase et al. (2014) presentaron una lista de 274 especies capturadas en diversos ambientes de la región interna del Golfo de Nicoya, desde la boca del Río Tempisque hasta frente al extremo del puerto de Puntarenas. De esas 274 especies, 188 (68 \%) fueron consideradas como habitantes de ambientes sedimentarios, como los 

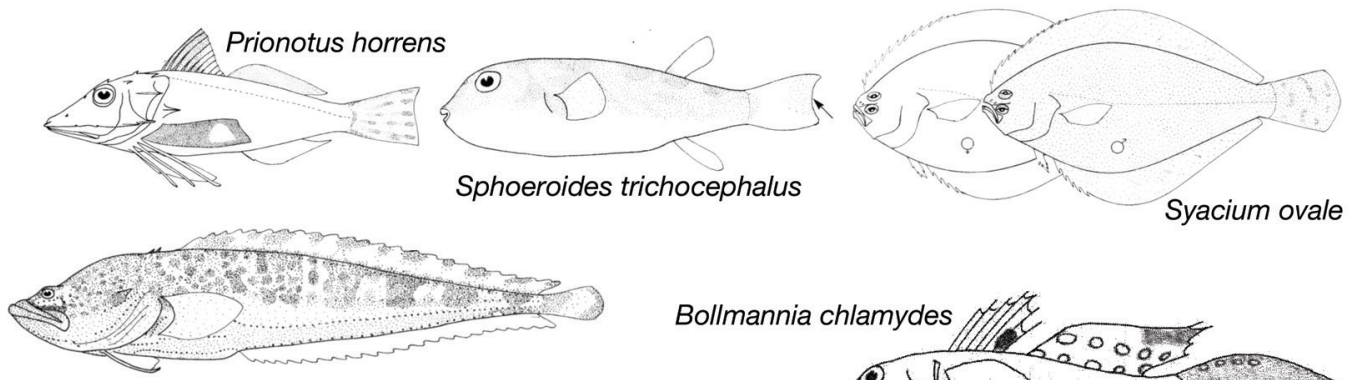

Bollmannia chlamydes
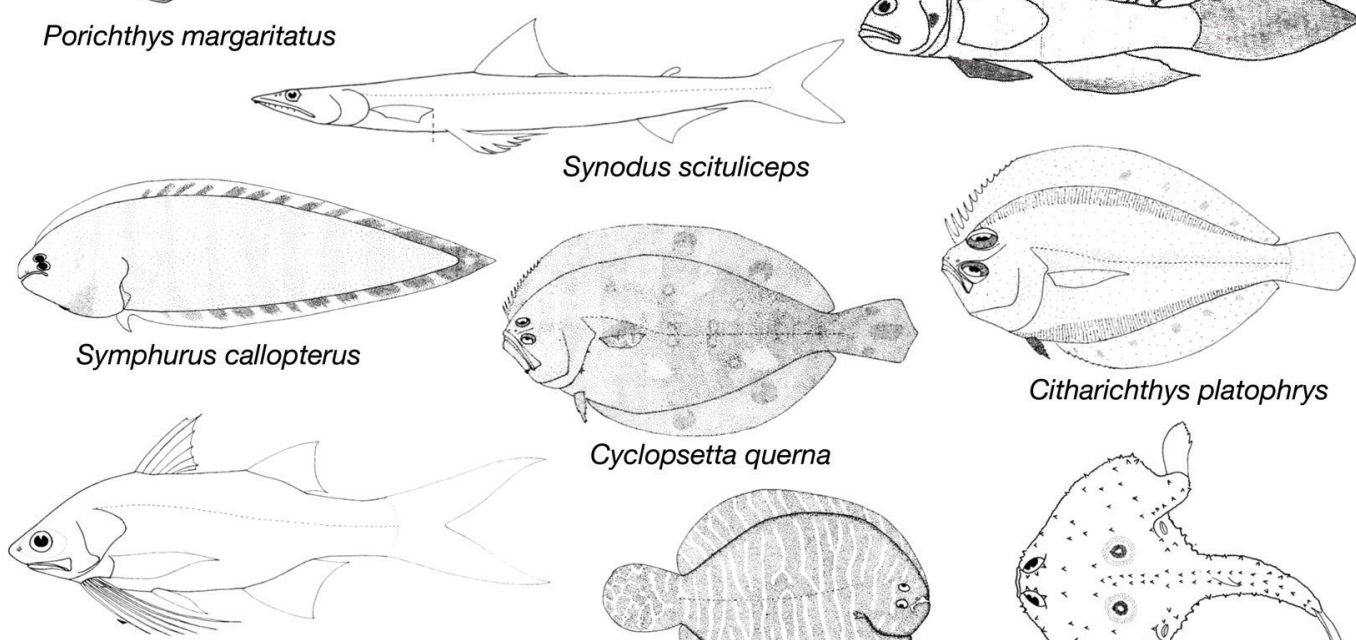

Cyclopsetta querna

Polydactylus approximans
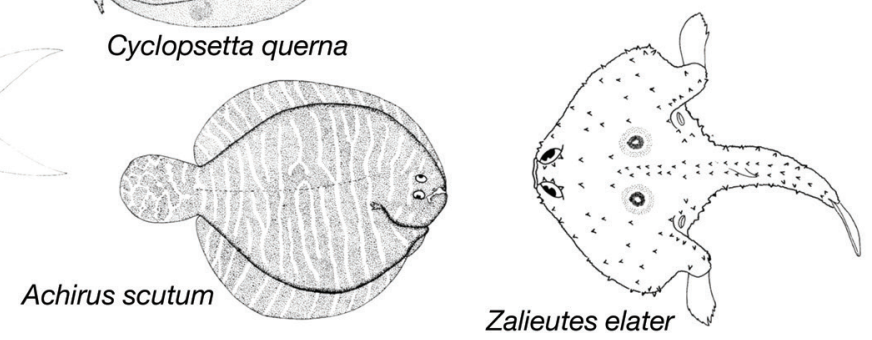

Fig. 3. Doce de las especies más frecuentes en ambas expediciones (Dibujos no a escala. De Bussing \& López, 2015).

Fig. 3. Twelve of the more frequent species in both expeditions (Drawings not to scale. From Bussing \& López, 2015).

estudiados durante los cruceros del Skimmer y V Hensen. En este contexto es importante anotar que las colecciones del MZUCR albergan ejemplares catalogados a partir de 1962 (Angulo et al., 2015) por lo que es necesario verificar la presencia actual de ejemplares en el estuario y el estado de sus poblaciones. Futuros estudios sobre la biodiversidad íctica del Golfo de Nicoya podrían incluir una comparación entre la presente lista y las aportadas por Erdman (1971), León (1973) y Murase et al., (2014). Las ocho especies descritas posteriormente a la publicación de la lista del V. Hensen (Bussing \& López, 1996) indican que el estuario todavía podría albergar algunas formas no conocidas, especialmente en aguas profundas. No obstante que la cobertura espacial del Golfo fue mayor durante la expedición del buque Skimmer (20 estaciones) que durante la del V. Hensen (12 estaciones) el uso en el V. Hensen de dos tipos de redes (otter y beam) y la toma de muestras a mayores profundidades (60 a $228 \mathrm{~m}$ ) permitió la captura de un mayor número de especies.

Dos de los tensores de mayor impacto en el estuario son la extracción de especies mediante redes de arrastre y el daño que esas artes de pesca causan a las comunidades infaunales y epifaunales propias de esos ambientes sedimentarios. Esos tensores son conocidos desde hace décadas (Jones, 1992) y para el Golfo de Nicoya el estudio de Rostad y Hansen (2001) ilustra la utilidad de preservar áreas vedadas a los arrastres, como ocurre para el área superior del estuario. Además, del total de los recursos 

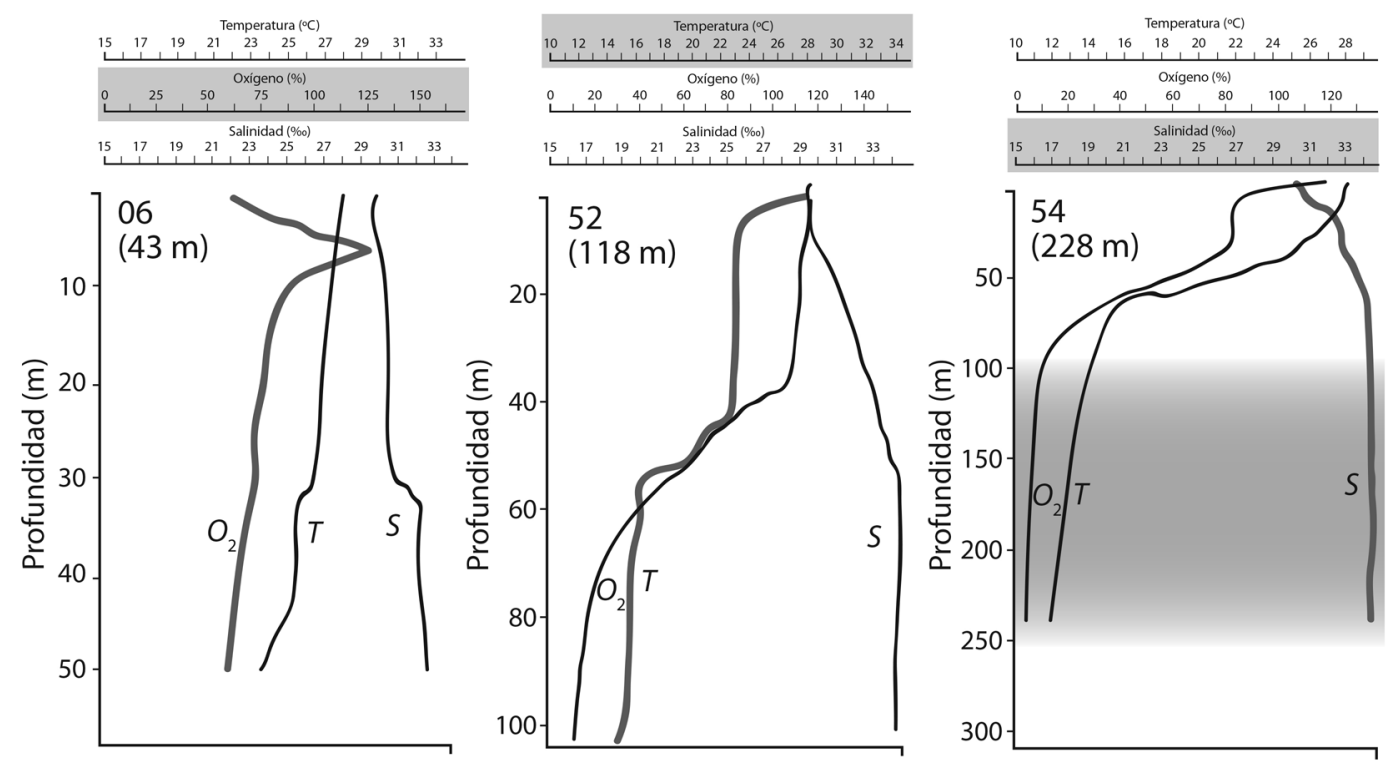

Fig. 4. Perfiles verticales (superficie - fondo ) obtenidos mediante sensor electrónico tipo CTD, de temperatura $\left(T\right.$, $\left.{ }^{\circ} \mathrm{C}\right), \%$ oxígeno disuelto $\left(\mathrm{O}_{2}\right)$ y salinidad $(S, \%$ ), el 3 y 4 de diciembre de 1993, en las estaciones 06 , 52, y 54 de la zona externa (boca) del Golfo de Nicoya, Pacífico, Costa Rica. La escala sombreada en gris (arriba) corresponde al perfil resaltado en negro abajo. El área gris (estación 54) representa el área, a profundidades mayores de $100 \mathrm{~m}$, de concentraciones de oxígeno menores del $5 \%$ de saturación. Modificada de Wolff y Vargas (1994, Fig. 1.1).

Fig. 4. Vertical profiles (surface - bottom) obtained by a CTD electronic sensor, of temperature $\left(T,{ }^{\circ} \mathrm{C}\right), \%$ dissolved oxygen $\left(\mathrm{O}_{2}\right)$ and salinity $(\mathrm{S}, \%$ ), on December 3 and 4, 1993, at the stations 06,52 and 54 on the exterior zone (mouth) of the Gulf of Nicoya, Pacific, Costa Rica. The gray shaded scale (above) corresponds to the black enhanced profile below. The gray shaded area (Station 54) represents the depth below $100 \mathrm{~m}$ of oxygen saturation below $5 \%$ Modified from Wolff \& Vargas (1994, Fig. 1.1).

(peces e invertebrados) capturados mediante redes solo se utiliza una fracción y el resto se descarta, por lo que desde hace tiempo se ha sugerido la posible utilización de algunas especies de peces descartados (Campos, 1983).

La presión de pesca sobre el grupo de peces cartilaginosos, como las rayas y tiburones, es importante en Costa Rica (Clarke, Espinoza, Romero-Chaves, \& Wehrtmann, 2017) y más de 15 especies están en riesgo y otras podrían estarlo en un futuro (Espinoza, Díaz, Angulo, Hernández, \& Clarke, 2018).

La mayoría de las especies de peces capturadas en los cruceros del Skimmer y del V. Hensen correspondió a peces óseos y es este el grupo que tradicionalmente ha recibido el mayor interés pesquero, especialmente las especies de las familias Sciaenidae (corvinas),
Serranidae (cabrillas y meros) y Lutjanidae (pargos). El impacto de la pesca de arrastre sobre otros grupos, que frecuentemente se descartan, como los lenguados y peces hoja (Bothidae, Cynoglossidae, Paralichthyidae y Achiridae) representados por 32 especies en los arrastres del Skimmer y V. Hensen en el Golfo de Nicoya, debe ser evaluado.

Según Campos, Burgos y Gamboa (1984) muchas de las especies capturadas por redes de arrastre en el Golfo de Nicoya en la época del Skimmer estaban representadas por individuos que no habían alcanzado la edad reproductiva. Por ejemplo, las longitudes esperadas a la primera madurez sexual de 15 especies de peces del Golfo, entre ellas las corvinas: Cynoscion albus $(35 \mathrm{~cm})$, C. phoxocephalus $(23.5 \mathrm{~cm}), C$. squamipinnis $(38 \mathrm{~cm})$, C. stolzmanni $(42.5 \mathrm{~cm})$ 
y Larimus acclivis $(21 \mathrm{~cm})$. Los ejemplares de mayor longitud de esas especies capturados en los cruceros del Skimmer y del V. Hensen fueron menores, con excepción de un ejemplar de C. albus $(75 \mathrm{~cm})$ obtenido por el V. Hensen y uno de L. acclivis $(34 \mathrm{~cm})$ del Skimmer. En futuros estudios se debe medir las longitudes de los peces para efectuar comparaciones con los datos históricos. En este contexto, es útil la información sobre longitudes totales incluida en los informes del Skimmer y del V. Hensen (Price et al., 1980; Wolff \& Vargas, 1994). Además, el estudio de Murase et al. (2014) aportó datos sobre las longitudes estándar de 274 especies de peces de la región interna del Golfo de Nicoya y depositados en la colección del MZUCR.

El análisis de la composición de especies de peces por estación recolectados por el V. Hensen dividió el Golfo en tres zonas: una interna, de aguas con profundidades menores a $50 \mathrm{~m}$; otra externa, con profundidades entre 100 y $240 \mathrm{~m}$, y una tercera zona de transición que incluye a las estaciones centrales y de los costados del estuario (Wolff, 1996). En los cruceros del Skimmer y del V. Hensen, como también en los hechos por León (1973) se encontraron dos tipos principales de distribución espacial de las especies de peces: aquellas con una amplia cobertura geográfica y otras presentes solo en ciertas estaciones, tal como lo ilustramos en este estudio. También, con base en la aparición de formas juveniles, fueron identificadas en esos cruceros zonas importantes del estuario para la reproducción de algunas especies. Esto fue reforzado con el hecho de que el estudio del ictioplancton en el Golfo de Nicoya en el crucero del V. Hensen permitió la identificación de larvas de peces pertenecientes a 16 familias (Molina-Ureña, 1996).

El Golfo de Nicoya ha continuado bajo la influencia cada vez mayor de la alteración de su entorno, así como de tensores regionales o globales, como El Niño-La Niña y el cambio climático. Por tanto, es urgente una nueva evaluación de los componentes del ecosistema a la luz de esos tensores. El enfoque ecosistémico ha sido propuesto como una alternativa al enfoque mono-especifico y busca un balance entre los servicios que presta el estuario y la salud del ecosistema (Jennings, 2004). En apoyo de ese enfoque, los cruceros del Skimmer y V. Hensen proporcionaron datos para la elaboración del primer modelo de Flujo Trófico del Golfo de Nicoya (FTGN), que permite estimar el impacto de la extracción, o adición, de componentes (biomasas de detritus, plancton, infauna, peces, crustáceos, moluscos, etc) al sistema (Wolff, Chavarría, Koch, \& Vargas, 1998; Vargas, 2016) La utilidad de este modelo, para evaluar cambios en las pesquerías del Golfo de Nicoya en consulta con los usuarios del sistema, ha sido enfatizado recientemente por Sánchez-Jiménez, Fujitani, MacMillan, Schluter y Wolff (2019).

En el contexto del flujo de energía del estuario, los peces de la familia Sciaenidae (corvinas), por ejemplo, son depredadores importantes en el ecosistema. En el estudio de León (1973), al menos ocho especies de corvinas (Cynoscion squamipinnis, Ophioscion scierus, O. typicus, Stellifer ericymba, S. fuerthii, S. illecebrosus, S. oscitans, y S. zestocarus) fueron capturadas en más del $50 \%$ de las 47 estaciones. Sin embargo, de esas ocho solo $S$. zestocarus aparece entre las más frecuentes en las 20 estaciones del Skimmer y ninguna entre las 12 del V. Hensen. Por lo tanto, debe ser evaluado el impacto de la remoción por pesca, de estos y otros peces depredadores en el ecosistema.

Entre los posibles cambios en el funcionamiento del Golfo de Nicoya, hay evidencia de que el estuario ha superado los valores de productividad primaria asociados a condiciones eutróficas (Cloern, Foster, \& Klechner 2014). Saturaciones de oxígeno superiores al $100 \%$, como la encontrada en la estación 06 del V. Hensen, además de concentraciones altas de clorofila- $a$ y estimaciones de productividad primaria neta superiores a $500 \mathrm{~g} \mathrm{C} / \mathrm{m}^{2} /$ año han sido documentadas en aguas superficiales del Golfo (Vargas-Zamora et al., 2018). Tal como lo ha indicado Jackson et al. (2001) la extracción de organismos que se alimentan de fitoplancton (como los moluscos bivalvos y algunos peces) ha contribuido a la eutroficación en algunos estuarios en otras latitudes. 
Los análisis de los parámetros físicos y químicos obtenidos por el buque Skimmer están disponibles en Voorhis, Epifanio, Maurer, Dittel y Vargas (1983) y en Epifanio, Maurer y Dittel (1983), respectivamente. Los parámetros ambientales recopilados para el Golfo de Nicoya por el V. Hensen fueron publicados por Chaves y Birkicht (1996). La gradiente de salinidad característica del estuario (Voorhis et al., 1983; Lizano \& Vargas, 1993), desde aguas salobres en la cercanía del Río Tempisque hasta salinidades propias de mar abierto en la región de la boca, es una barrera importante en la distribución de los peces, tal como lo evidenció el estudio de León (1973). Las observaciones del V. Hensen indican que, en las zonas media e inferior del estuario, otras gradientes se acentúan a profundidades mayores de $\operatorname{los} 50 \mathrm{~m}$, con temperaturas inferiores a $\operatorname{los} 12{ }^{\circ} \mathrm{C}$ y concentraciones de oxígeno inferiores al $5 \%$ de saturación (Wolff, 1996). Los ambientes de baja temperatura e hipóxicos son característicos de grandes regiones del Pacífico Este Tropical y para algunas especies de peces estuarinas esas condiciones podrían representar barreras en su distribución (Prince \& Goodyear, 2006).

Uno de los tensores del Golfo de Nicoya es el ingreso de contaminantes acarreados en gran parte por los ríos que desembocan en el estuario (Fuller et al., 1990; Spongberg 2004; García, Acuña-González, Vargas-Zamora, \& GarcíaCéspedes, 2006; Spongberg et al., 2011). Algunos de estos contaminantes podrían influir en la capacidad reproductiva de especies de peces e invertebrados través de la disrupción endocrina (Cheek, 2006; Lee et al., 2005; Gravel, Johanning, McLachlan, Vargas, \& Oberdörster, 2006). La presencia combinada de hipoxia y de disrupción endocrina ha sido informada como un factor que podría ser importante en la reproducción de algunos peces estuarinos (Thomas, Rahman, Khan, \& Kummer, 2007).

En este contexto complejo, las preguntas más urgentes de responder sobre la ictiofauna del Golfo de Nicoya, son: ¿Cuántas especies de peces integran actualmente la fauna del estuario? y ¿Cuáles estrategias de manejo del ecosistema podrían contribuir a su sobrevivencia?
Este estudio complementa el publicado por Vargas-Zamora, Vargas-Castillo y SibajaCordero (2019) sobre los crustáceos (Decapoda y Stomatopoda) capturados por los buques Skimmer y V. Hensen en el Golfo de Nicoya.

Declaración de ética: los autores declaran que todos están de acuerdo con esta publicación y que han hecho aportes que justifican su autoría; que no hay conflicto de interés de ningún tipo; y que han cumplido con todos los requisitos y procedimientos éticos y legales pertinentes. Todas las fuentes de financiamiento se detallan plena y claramente en la sección de agradecimientos. El respectivo documento legal firmado se encuentra en los archivos de la revista.

\section{AGRADECIMIENTOS}

Este trabajo está dedicado al recuerdo del Prof. William Bussing Burhaus (1933-2014), ictiólogo, co-fundador en la Universidad de Costa Rica, del Museo de Zoología (MZUCR) y del Centro de Investigación en Ciencias del Mar y Limnología (CIMAR). Esta publicación conmemora el 40 aniversario (1979-2019) de la fundación del CIMAR. Los cruceros del buque Skimmer fueron parte del Convenio de Cooperación entre la Universidad de Costa Rica - CIMAR y la Universidad de Delaware - Colegio de Estudios Marinos (CMS), Lewes, E.E.U.U. Los cruceros del buque Víctor Hensen fueron parte del Convenio de Cooperación entre la Universidad de Costa Rica - CIMAR y el Centro de Ecología Marina Tropical (ZMT), Bremen, Alemania. A cuatro revisores externos por sus comentarios y referencias. A Sergio Aguilar por la preparación de las tablas y figuras.

\section{RESUMEN}

Introducción: Desde mediados del siglo XX el estuario del Golfo de Nicoya $\left(10^{\circ} \mathrm{N} \& 85^{\circ} \mathrm{W}\right)$ ha sido la fuente principal de numerosos recursos pesqueros, como los moluscos, crustáceos y peces. El estuario está actualmente bajo impactos crecientes locales, regionales y globales. Las 
expediciones a bordo de los buques científicos Skimmer (1979-1980) y Víctor Hensen (1993-1994) aportaron datos multidisciplinarios en apoyo de políticas de manejo. Sin embargo, actualmente se necesita información actualizada. Objetivos: Actualizar las listas publicadas de peces recolectados en ambas expediciones y sugerir nuevas áreas de investigación para una futura evaluación del estado del ecosistema. Métodos: En el buque Skimmer las capturas de peces cubrieron un ámbito de profundidad de 10 a $50 \mathrm{~m}$, con una red tipo otter. En el buque V. Hensen las capturas se hicieron entre 10 y $228 \mathrm{~m}$ de profundidad, con redes tipo otter y beam. En ambos cruceros se hizo a bordo la identificación de la mayoría de las especies y la medición de sus longitudes totales. Para actualizar las listas de especies se procedió a la verificación de los nombres vigentes con base en literatura reciente y la página World Register of Marine Species (WORMS). Agregamos a la lista los nombres de las especies identificadas en años recientes para el estuario y catalogados en la Colección Ictiológica del Museo de Zoología, Universidad de Costa Rica. Resultados: El total de especies identificadas es de 268 para ambos buques. Una especie de Pontinus esta en proceso de descripción. Del total, 19 especies corresponden a peces cartilaginosos y 249 a peces óseos. Los Sciaenidae (corvinas) y los lenguados estuvieron representados por 31 y 32 especies, respectivamente. El género Symphurus (lenguados) fue el más diverso, con 11 especies. Dieciocho especies fueron identificadas después de la publicación de las listas originales. Ocho especies fueron descritas con base en ejemplares recolectados por ambos buques: Gymnothorax phalarus, Notarius biffi, N. cookei, Ophicthus mecoptera, O. melope, Sphoeroides rosenblatti, Trinectes xanthurus y Urotrygon cimar. Los cinco peces de mayor longitud capturados por ambos buques, fueron: la raya látigo Hypanus longus (117 $\mathrm{cm})$, el congrio Cynoponticus coniceps $(103 \mathrm{~cm})$, el bagre Sciades dowii $(87 \mathrm{~cm})$, la raya Narcine brancroftii $(80 \mathrm{~cm})$ y la corvina reina Cynoscion albus $(75 \mathrm{~cm})$. Conclusiones: En vista del incremento del número de usuarios de los recursos estuarinos, es urgente una evaluación multidisciplinaria del ecosistema en apoyo a la propuesta de nuevas políticas de manejo. Los resultados facilitarían actualizar el Modelo Trófico del Golfo de Nicoya, una herramienta de manejo disponible desde 1998 y basada principalmente en datos obtenidos durante los cruceros del Skimmer y V. Hensen. Tensores del ecosistema, como la extracción de recursos, el ingreso de contaminantes y su posible relación con la disrupción endocrina, así como la presencia de barreras naturales como la hipoxia en la boca del estuario, deben ser incluidos en esa futura evaluación.

Palabras clave: peces estuarinos; pesquerías; longitud de peces; redes de arrastre; bentos; Prionotus; Porichthys; Symphurus.

\section{REFERENCIAS}

Angulo, A., López, M., Bussing, W., Ramírez-Coghi, A. R., \& Arias-Godínez, G. (2015). Colección ictiológica del Museo de Zoología de la Universidad de Costa Rica. In L. F. Del Moral-Flores, A. J. Ramírez-Villalobos, J. A. Martínez-Pérez, A. F. González-Acosta \& J. Franco-López (Eds.), Colecciones Ictiológicas de Latinoamérica (pp. 56-65). México: Facultad de Estudios Superiores Iztacala, Universidad Nacional Autónoma de México \& Sociedad Mexicana de Ictiología.

Bartels, C., Price, K. S., López, M., \& Bussing, W. A. (1983). Occurrence, distribution, abundance and diversity of fishes in the Gulf of Nicoya, Costa Rica. Revista de Biología Tropical, 31, 75-101.

Bartels, C., Price, K. S., López-Bussing, M., \& Bussing, W. A. (1984). Ecological assessment of finfish as indicators of habitats in the Gulf of Nicoya, Costa Rica. Hydrobiologia, 112, 197-207.

Bearez, P. (1996). Lista de los peces marinos del Ecuador continental. Revista de Biología Tropical, 44, 731-741.

Bussing, W. A., \& López, M. I. (1993). Peces demersales y pelágicos costeros del Pacífico de Centro América Meridional. Guía Ilustrada / Demersal and Pelagic Inshore Fishes of the Pacific Coast of Lower Central America. An Illustrated Guide. Revista de Biología Tropical, 41(Publicación Especial - Special Publication), 1-164.

Bussing, W. A., \& López, M. I. (1996). Fishes collected during the R.V. Victor Hensen Costa Rica Expedition (1993/1994). Revista de Biología Tropical, 44(Supplement 3), S183-S186.

Bussing, W. A., \& López, M. (2009). Marine fish. In I. S. Wehrtmann \& J. Cortés (Eds.), Marine Biodiversity of Costa Rica, Central America (Text: pp. 453-458, List of species: CD disk pp. 412-473). Monographiae Biologicae 86. Berlin: Springer + Business Media B.V.

Bussing, W. A., \& López, M. I. (2015). Peces demersales y pelágicos costeros del Pacifico de Centroamérica Meridional. / Demersal and pelagic fishes of the Pacific coast of lower Central America. San José: Editorial de la Universidad de Costa Rica.

Campos, J. (1983). Talla de los peces descartados de la fauna de acompañamiento del camarón como un indicador de su posible utilización. Revista de Biología Tropical, 31, 209-212.

Campos, J., Burgos, B., \& Gamboa, C. (1984). Effect of shrimp trawling on the commercial ichthyofauna of the Gulf of Nicoya, Costa Rica. Revista de Biología Tropical, 32, 203-207. 
Castellanos-Galindo, C. A., Rubio-Rincón, E. A., BeltránLeón, B., Zapata, L. A., \& Baldwin, C. C. (2006). Peces marinos de los órdenes Gadiformes, Ophidiiformes y Lophiiformes en aguas colombianas del Pacífico oriental tropical. Biota Colombiana, 7(2), 191-209.

Chaves, J., \& Birkicht, M. (1996). Equatorial Subsurface Water and the nutrient seasonality distribution of the Gulf of Nicoya, Costa Rica. Revista de Biología Tropical, 44(Supplement 3), S41-S47.

Cheek, A. O. (2006). Subtle sabotage: endocrine disruption in wild populations. Revista de Biología Tropical, 54(Supplement 1), S1-S19.

Clarke, T. M., Espinoza, M., Romero-Chaves, R., \& Wehrtmann, I. S. (2017). Assessing the vulnerability of demersal elasmobranchs to a data-poor shrimp trawl fishery in Costa Rica, Eastern Tropical Pacific. Biological Conservation, 217, 321-328.

Cloern, J. E., Foster, S. O., \& Klechner, A. E. (2014). Phytoplankton primary production in the world's estuarine-coastal ecosystems. Biogeosciences, 11, $2477-2501$

Cloern, J. E., Abreu, P., Carstensen, J., Chauvaud, L., Elmgren, R., Grall, J., ... Yin, K. (2016). Human activities and climate variability drive fast-paced change across the world's estuarine-coastal ecosystems. Global Change Biology, 22, 513-529.

Edrman, D. S. (1971). Notes on the fishes from the Gulf of Nicoya, Costa Rica. Revista de Biología Tropical, 19, 59-71.

Epifanio, C. E., Maurer, D., \& Dittel, A. I. (1983). Seasonal changes in nutrients and dissolved oxygen in the Gulf of Nicoya, a tropical estuary on the Pacific coast of Central America. Hydrobiologia, 101, 231-238.

Espinoza, M., Díaz, E., Angulo, A., Hernández, S., \& Clarke, T. M. (2018). Chondrichthyan diversity, conservation status, and management challenges in Costa Rica. Frontiers in Marine Science, 5, 85.

Fuller, C. C., Davis, J. A., Cain, D. J., Lamothe, P. J., Fries, T. L., Fernández, G., ... Murillo, M. M. (1990). Distribution and transport of sediment-bound metal contaminants in the Río Grande de Tárcoles, Costa Rica (Central America). Water Research, 24, 805-812.

García, V., Acuña-González, J., Vargas-Zamora, J. A., \& García-Céspedes, J. (2006). Calidad bacteriológica y desechos sólidos en cinco ambientes costeros de Costa Rica. Revista de Biología Tropical, 54(Suplemento 1), S35-S48.

Gladstone, W. (2009). Conservation and management of coastal tropical ecosystems. In I. Nagelkerden (Ed.), Ecological Connectivity among Tropicial Coastal Ecosysems (pp. 565-605). Berlin: Springer + Business Media B.V.

Gravel, P., Johanning, K., McLachlan, J., Vargas, J. A., \& Oberdörster, E. (2006). Imposex in the intertidal snail Thais brevidentata (Gastropoda: Muricidae) from the Pacific coast of Costa Rica. Revista de Biología Tropical, 54(Supplement 1), S21-S26.

Herrera, M., Clarke, T. M., Naranjo-Elizondo, B., Espinoza, M., \& Wehrtmann, I. S. (2016). Size at maturity of the Pacific bearded brotula (Ophidiidae: Brotula clarkae): a commercially exploited species in the Pacific of Costa Rica. Latin American Journal of Aquatic Research, 44, 657-661.

Jackson, J. B. C., Kirby, M. X., Berger, W. H., Bjondal, K., Botsford, L. W., Bourger, B. C., ... Warner, R. R. (2001). Historical overfishing and the recent collapse of coastal ecosystems. Science, 293, 629-638.

Jennings, S. (2004). The ecosystem approach to fishery management: a significant step towards sustainable use of the marine environment. Marine Ecology Progress Series, 274, 269-303.

Jones, J. B. (1992). Environmental impact of trawling on the sea bed: A review. New Zealand Journal of Marine and Freshwater Research, 26, 59-67.

Lee, J., Johanning, K., Cheek, A., Vargas, J. A., Bonilla, J., \& McLachlan, J. (2005). Utilizing the spottedrose snapper (Lutjanus guttatus) as an indicador of endocrine disrupting chemicals in the Pacific coast of Costa Rica. International Workshop on Crop Protection Chemistry in Latin America. San José, Costa Rica: IUPAC-CICA/UCR, SFE-MAG.

León, P. E. (1973). Ecología de la ictiofauna del Golfo de Nicoya, Costa Rica, un estuario tropical. Revista de Biología Tropical, 21, 5-30.

Lizano, O., \& Vargas, J. A. (1993). Distribución espaciotemporal de la salinidad y la temperatura en la parte interna del Golfo de Nicoya. Tecnología en Marcha, 12(2), 3-16.

Molina-Ureña, H. (1996). Ichthyoplankton assemblages in the Gulf of Nicoya and Golfo Dulce embayments, Pacific coast of Costa Rica. Revista de Biología Tropical, 44(Supplement 3), S173-S182.

Murase, A., Angulo, A., Miyasaki, Y., Bussing, W. A., \& López, M. I. (2014). Marine and estuarine fish diversity in the inner Gulf of Nicoya, Pacific coast of Costa Rica, Central America. Check List, 10, 1401-1413.

Price, K., Bussing, M., Bussing, W. A., Maurer, D., \& Bartels, C. (1980). Finfish survey. In D. Maurer, C. Epifanio, \& K. Price (Eds.), Ecological assessment of finfish and megabenthic invertebrates as indicators of natural and impacted habitats in the Gulf of Nicoya, Costa Rica (pp. 83-144). Newark, Delaware, U.S.A.: University of Delaware, College of Marine Studies, Progress Report of the 1979 International Sea Grant Program.

Prince, E. D., \& Goodyear, C. P. (2006). Hypoxia-based habitat compression of tropical pelagic fishes. Fisheries Oceanography, 15, 451-464.

Rostad, T., \& Hansen, K. L. (2001). The effects of trawling on the benthic fauna of the Gulf of Nicoya, Costa 
Rica. Revista de Biología Tropical, 49(Supplement 2), S91-S95.

Sánchez-Jiménez, A., Fujitani, M., MacMillan, D., Schluter, A., \& Wolff, M. (2019). Connecting a Trophic Model and local knowledge to improve fisheries management: The case of the Gulf of Nicoya, Costa Rica. Frontiers in Marine Science, 6, 126.

Smith, D. (2018). Fishes collected by Captain John M. Dow mainly on the Pacific coast of Central America (1861-1865) and deposited in the National Museum of Natural History, Smithsonian Institution. Proceedings of the Biological Society of Washington, 131, 65-89.

Spongberg, A. L. (2004). PCB concentrations in sediments from the Gulf of Nicoya estuary, Pacific coast of Costa Rica. Revista de Biología Tropical, 52(Supplement 2), S11-S22.

Spongberg, A. L., Witter, J. D., Acuña, J., Vargas, J., Muri1lo, M., Umaña, G., \& Pérez, G. (2011). Reconnaissance of selected PPCP compounds in Costa Rican surface waters. Water Research, 45, 6709-6717.

Thomas, P., Rahman, M. S., Khan, I. A., \& Kummer, J. A. (2007). Widespread endocrine disruption and reproductive impairment in an estuarine fish population exposed to seasonal hypoxia. Proceedings of the Royal Society B., 274, 2693-2701.

Vargas, J. A. (1995). The Gulf of Nicoya estuary, Costa Rica: Past, present, and future cooperative research. Helgolander Meeresuntersuchungen, 49, 821-828.

Vargas, J. A. (2016). The Gulf of Nicoya estuarine ecosystem. In M. Kappelle (Ed.) Costa Rican Ecoystems (pp 139-161). Chicago. University of Chicago Press.
Vargas, J. A., \& Wolff, M. (Eds.). (1996). Ecosistemas Costeros de Costa Rica, con énfasis en el Golfo Dulce y áreas adyacentes: una visión sinóptica basada en la expedición del B. O. Victor Hensen 1993/1994 y estudios previos. Revista de Biología Tropical, 44(Suplemento 3), S1-S238.

Vargas-Zamora, J. A., Acuña-González, J., Sibaja-Cordero, J. A., Gómez-Ramírez, E. H., Agüero-Alfaro, G., \& García-Céspedes, J. (2018). Water parameters and primary productivity at four coastal embayments of Costa Rica (2000-2002). Revista de Biología Tropical, 66(Supplement 1), S211-S230.

Vargas-Zamora, J. A., Vargas-Castillo, R., \& Sibaja-Cordero, J. A. (2019). Crustáceos (Decapoda y Stomatopoda) del R.V. Skimmer y R.V. Victor Hensen en el Golfo de Nicoya, Pacífico, Costa Rica. Revista de Biología Tropical, 67, 286-305.

Voorhis, A., Epifanio, C. E., Maurer, D., Dittel, A. I., \& Vargas, J. A. (1983). The estuarine character of the Gulf of Nicoya, an embayment on the Pacific coast of Central America. Hydrobiologia, 99, 225-237.

Wolff, M. (1996). Demersal fish assemblages along the Pacific coast of Costa Rica: a quantitative and multivariate assessment based on the Victor Hensen Costa Rica Expediton 1993/1994. Revista de Biología Tropical, 44(Supplement 3), S187-S214.

Wolff, M., Chavarría, J., Koch, V., \& Vargas, J. A. (1998). A trophic flow model of the Golfo de Nicoya, Costa Rica. Revista de Biología Tropical, 46(Supplement 6), 63-79.

Wolff, M., \& Vargas, J. A. (Eds.). (1994). RV Victor Hensen Costa Rica Expedition 1993 / 1994 Cruise Report. Bremen, Germany. ZMT Contributions, 2, 1-109. 